\title{
Synthesis and Antimicrobial Studies on New Substituted 1,3,4-Oxadiazole Derivatives Bearing 6-Bromonaphthalene Moiety
}

\author{
Anil N. Mayekar \\ Department of Studies in Chemistry, University of Mysore \\ Manasagangotri 570 006, India \\ SeQuent Scientific Limited, 120 A and B, Industrial Area \\ Baikampady, New Mangalore, 575 011, India \\ Tel: 91-824-2402306 E-mail: anilmayekar@gmail.com \\ H. S. Yathirajan (Corresponding author) \\ Department of Studies in Chemistry, University of Mysore \\ Manasagangotri 570 006, India \\ Tel: 91-821-2419656 \\ E-mail: yathirajan@hotmail.com \\ B. Narayana \\ Department of Chemistry, Mangalore University \\ Mangalagangotri 574 199, India \\ Tel: 91-824-2287262Ｅ-mail: nbadiadka@yahoo.co.uk \\ B. K. Sarojini \\ Department of Chemistry, P. A. College of Engineering Nadupadavu \\ Mangalore 574 153, India \\ Tel: 91-824-2284701Ｅ-mail: bksaroj@yahoo.com \\ N. Suchetha Kumari \\ Department of Biochemistry, Justice K.S. Hegde Medical Academy \\ Deralakatte, Mangalore 574 162, India \\ Tel: 91-824-2202471Ｅ-mail: suchetha.shetty@rediffmail.com
}

This research work is supported by University of Mysore, Mysore and SeQuent Scientific Ltd., Mangalore

\begin{abstract}
A series of new 1,3,4-oxadiazole derivatives having 6-bromonaphthalene moiety are synthesized. 2-[(6-bromo-2-naphthyl)oxy]acetohydrazide was treated with various substituted aromatic acids in presence of $\mathrm{POCl}_{3}$ to give 2-\{[(6-bromo-2-naphthyl)oxy]methyl $\}-5$-aryl-1,3,4-oxadiazole. Also the hydrazide on treating with $\mathrm{CS}_{2} / \mathrm{KOH}$ gave 5-\{[(6-bromo-2-naphthyl)oxy]methyl $\}-1,3,4$-oxadiazole-2(3H)-thione, which was subjected to Mannich reaction to get a series of Mannich bases and with alkyl/aryl halide to give 2-\{[(6-bromo-2-naphthyl)oxy]methyl\}-5-[(alkyl/aryl)thio]-1,3,4-oxadiazole. The newly synthesized compounds were characterized by analytical and spectral data. Antimicrobial activities of these compounds were carried out and some of them have exhibited good activity.
\end{abstract}


Keywords: 1,3,4-Oxadiazole-2(3H)-thione, Mannich base,

2-\{[(6-Bromo-2-naphthyl)oxy]methyl\}-5-aryl-1,3,4-oxadiazole,

2-\{[(6-Bromo-2-naphthyl)oxy]methyl\}-5-[(alkyl/aryl)thio]-1,3,4-oxadiazole, Antimicrobial activity

\section{Introduction}

1,3,4-Oxadiazoles are heterocyclic compounds, which serve both as biomimetic and reactive pharmacophores and many are the key compounds with potential biological activities (Hokfelt et al., 1962; Hashem et al., 2007; Boschelli et al., 1993; Adelstein et al., 1976). For instance, 2-amino-1,3,4-oxadiazole acts as muscle relaxtant (Yale et al., 1966) and 1,3,4-oxadiazole having naphthalene nucleus have shown to posses anti-inflammatory activity (Amir et al., 1998; Rajak et al., 2007). Synthesis, anti-inflammatory and antiproteolytic properties of naphthyl thio semicarbazides and cyclized oxadiazoles derivatives have been reported (Kishore et al., 1975). 1,3,4-oxadiazole derivatives are good monoamine oxidase inhibitors (antidepressants), anti-convulsant agent (Shaoyong et al., 2008; Zarghi et al., 2008) and potential inhibitors targeting chitin biosynthesis (Shaoyong et al., 2009).

In recent years, Mannich bases have gained importance because of their pharmaceutical importance (Dimmock et al., 1997; Sridhar et al., 2002). The presence of basic Mannich side chain has shown a marked antimalarial activity (Kotecka et al., 1997). Studies revealed that Mannich bases showed good anticancer (Aboraia et al., 2005) and antimycobacterial activity (Ali et al., 2007). Mannich bases are also known to posses remarkable anti-HIV and antitubercular activities (Sriram et al., 2009). Also 1,3,4-oxadiazole-5-thioether derivatives have been the object of investigation due to their different properties such as antibacterial (Macaev et al., 2005) and antifungal (Chen et al., 2000). In continuation of our work on 1,3,4-oxadiazole derivatives bearing naphthalene ring (Narayana et al., 2005) it was contemplated to synthesize some new 1,3,4-oxadiazole derivatives bearing 6-bromonaphthalene moiety.

\section{Material and Methods}

\subsection{Experimental}

TLC was run on a Merck silica gel 60 F254 coated aluminum plates and melting points were taken in open capillary tubes and are uncorrected. Elemental analysis was carried out using Flash EA 1112 Series, CHNSO Analyzer (Thermo). IR spectra in $\mathrm{KBr}$ pellets were recorded on Jasco FT/IR-4100 FTIR spectrophotometer. ${ }^{1} \mathrm{H}-\mathrm{NMR}$ spectra were recorded in $\mathrm{CDCl}_{3}$ and in DMSO- $\mathrm{d}_{6}$ on a Bruker DRX-300 (300 MHz) spectrometer using TMS as internal standard and Mass spectra were recorded on a Jeol SX 102/Da-600 mass spectrometer/Data System using Argon/Xenon (6 kV,10 mA) as FAB gas.

\subsubsection{General procedure for the preparation of ethyl (6-bromo-2-naphthyloxy) acetate (2)}

6-Bromo-2-naphthol (1) (10 g, $0.044 \mathrm{~mol})$ was dissolved in $250 \mathrm{ml}$ dry acetone and mixed with anhydrous potassium carbonate $(11 \mathrm{~g}, 0.08 \mathrm{~mol})$. This was treated with ethylchloroacetate $(5.4 \mathrm{~g}, 0.044 \mathrm{~mol})$ and the mixture was refluxed for 5-6 h. After the completion of reaction (monitored by TLC), the reaction mixture was filtered and the filtrate was distilled under reduced pressure. The solid obtained was recrystallized from acetonitrile. Yield $88 \%$, m.p. $62-65^{\circ} \mathrm{C}$.

The structure of the compound was confirmed from single crystal X-ray study (Sarojini, Yathirajan et al., 2007).

\subsubsection{General procedure for the preparation of 2-[(6-bromo-2-naphthyl)oxy]acetohydrazide (3)}

A mixture of ethyl(6-bromo-2-naphthyloxy)acetate (2) $(5.0 \mathrm{~g}, 0.01 \mathrm{~mol})$ and hydrazine hydrate $(1.6 \mathrm{~g}, 0.02 \mathrm{~mol})$ in ethanol $(20 \mathrm{ml})$ was heated under reflux for $5 \mathrm{~h}$. The reaction mixture was cooled to room temperature and the solid separated was collected by filteration. It was washed with ethanol and recrystallized in methanol. The product was obtained as light yellow solid in $92 \%$ yield, m.p $198-200{ }^{\circ} \mathrm{C}$.

Elemental analysis \% Calculated (Found) for $\mathrm{C}_{12} \mathrm{H}_{11} \mathrm{BrN}_{2} \mathrm{O}_{2:} \mathrm{C}-48.83(48.79)$ ), $\mathrm{H}-3.75(3.71)$ and N-9.49(9.43). IR ( $\mathrm{KBr}$, $\left.\gamma_{\max } \mathrm{cm}^{-1}\right): 3311\left(\mathrm{NH}_{2}\right), 3206(\mathrm{NH}), 1667(\mathrm{C}=\mathrm{O}), 1541(\mathrm{C}=\mathrm{C}) .{ }^{1} \mathrm{H}$ NMR (DMSO-d 6 , $\delta$ ppm): 4.35(bs, 2H, NH $\left.\mathrm{NH}_{2}\right), 4.60(\mathrm{~s}$, $\left.2 \mathrm{H}, \mathrm{CH}_{2}\right), 7.28-7.32(\mathrm{~m}, 2 \mathrm{H}$, naphthalene ring proton), $7.57(\mathrm{~d}, \mathrm{~J}=6.5 \mathrm{~Hz}, 1 \mathrm{H}$, naphthalene ring proton), $7.75(\mathrm{~d}, \mathrm{~J}=6.5$ $\mathrm{Hz}, 1 \mathrm{H}$, naphthalene ring proton), $7.84(\mathrm{~d}, \mathrm{~J}=6.5 \mathrm{~Hz}, 1 \mathrm{H}$, naphthalene ring proton), $8.11(\mathrm{~s}, 1 \mathrm{H}$, naphthalene ring proton), 9.41 (bs, $1 \mathrm{H}, \mathrm{NH})$. $\mathrm{MS} \mathrm{FAB}^{+}(\%): 295(100) \mathrm{M}^{+}, 297(85)[\mathrm{M}+2]^{+}$.

2.1.3 Procedure for the preparation of 2-\{[(6-bromo-2-naphthyl)oxy]methyl $\}$-5-aryl-1,3,4-oxadiazole (4a-r).

2-[(6-Bromo-2-naphthyl)oxy]acetohydrazide (1 g, $0.003 \mathrm{~mol})$ was refluxed with (un)substituted aromatic acid $(0.003$ $\mathrm{mol})$ in $\mathrm{POCl}_{3}(5 \mathrm{ml})$ for $8 \mathrm{~h}$. The reaction mixture was slowly quenched into crushed ice with stirring and neutralized it with solid sodium bicarbonate. The solid which separated after standing overnight was filtered, washed with cold water, dried, and recrystallized from methanol to afford the title compounds. The characterization data are given in Table-1. The spectral details are given below.

2-\{[(6-Bromo-2-naphthyl)oxy]methyl\}-5-(2-methoxyphenyl)-1,3,4-oxadiazole (4a)

IR (KBr, $\left.\gamma_{\max } \mathrm{cm}^{-1}\right)$ : 2935 (C-H str), $1640(\mathrm{C}=\mathrm{N}), 1234(\mathrm{C}-\mathrm{O})$. 
${ }^{1} \mathrm{H} \mathrm{NMR}\left(\mathrm{CDCl}_{3}, \delta \mathrm{ppm}\right): 3.93\left(\mathrm{~s}, 3 \mathrm{H},-\mathrm{OCH}_{3}\right), 5.45\left(\mathrm{~s}, 2 \mathrm{H}, \mathrm{O}-\mathrm{CH}_{2}\right) 7.06$ (t, J=8.8 Hz, 2H, phenyl ring proton), 7.27 (d, $\mathrm{J}=8.5 \mathrm{~Hz}, 1 \mathrm{H}$, naphthalene ring proton), $7.32(\mathrm{~s}, 1 \mathrm{H}$, naphthalene ring proton), $7.49(\mathrm{~d}, \mathrm{~J}=7.2 \mathrm{~Hz}, 1 \mathrm{H}$, phenyl ring protons), 7.53 (d, J=8.6 Hz, 1H, naphthalene ring proton), $7.64(\mathrm{~d}, \mathrm{~J}=8.8 \mathrm{~Hz}, 1 \mathrm{H}$, naphthalene ring proton), 7.69 (d, $\mathrm{J}=8.9 \mathrm{~Hz}, 1 \mathrm{H}$, naphthalene ring proton), $7.93(\mathrm{~s}, 1 \mathrm{H}$, naphthalene ring proton), $7.95(\mathrm{~d}, \mathrm{~J}=7.8 \mathrm{~Hz}, 1 \mathrm{H}$, phenyl ring protons). $\mathrm{MS} \mathrm{FAB}^{+}(\%): 411(90 \%) \mathrm{M}^{+}, 413(100 \%)[\mathrm{M}+2]^{+}$.

2-\{[(6-Bromo-2-naphthyl)oxy]methyl\}-5-(2-fluorophenyl)-1,3,4-oxadiazole (4b)

IR $\left(\mathrm{KBr}, \gamma_{\max } \mathrm{cm}^{-1}\right): 3035(\mathrm{C}-\mathrm{H}$ str), $1648(\mathrm{C}=\mathrm{N}), 1262(\mathrm{C}-\mathrm{O})$.

${ }^{1} \mathrm{H}$ NMR $\left(\mathrm{CDCl}_{3}, \delta \mathrm{ppm}\right): 5.47$ (s, 2H, $\left.\mathrm{O}-\mathrm{CH}_{2}\right)$ 7.23-7.33 (m, 3H, phenyl ring proton and $2 \mathrm{H}$, naphthalene ring proton), $7.54(\mathrm{~d}, \mathrm{~J}=8.3 \mathrm{~Hz}, 1 \mathrm{H}$, naphthalene ring proton), $7.65(\mathrm{~d}, \mathrm{~J}=8.6 \mathrm{~Hz}, 1 \mathrm{H}$, naphthalene ring proton), $7.70(\mathrm{~d}, \mathrm{~J}=8.8 \mathrm{~Hz}, 1 \mathrm{H}$, naphthalene ring proton), $7.94(\mathrm{~s}, 1 \mathrm{H}$, naphthalene ring proton), $8.0(\mathrm{t}, \mathrm{J}=7.8 \mathrm{~Hz}, 1 \mathrm{H}$, phenyl ring protons). MS $\mathrm{FAB}^{+}(\%): 399(85 \%) \mathrm{M}^{+}, 401(100 \%)[\mathrm{M}+2]^{+}$.

2-\{[(6-Bromo-2-naphthyl)oxy]methyl\}-5-phenyl-1,3,4-oxadiazole (4c)

IR (KBr, $\left.\gamma_{\max } \mathrm{cm}^{-1}\right): 2931$ (C-H str), $1644(\mathrm{C}=\mathrm{N}), 1034(\mathrm{C}-\mathrm{O})$.

${ }^{1} \mathrm{H}$ NMR $\left(\mathrm{CDCl}_{3}, \delta \mathrm{ppm}\right): 5.44\left(\mathrm{~s}, 2 \mathrm{H}, \mathrm{O}-\mathrm{CH}_{2}\right), 7.24(\mathrm{~d}, \mathrm{~J}=6.4 \mathrm{~Hz}, 1 \mathrm{H}$, naphthalene ring proton), $7.31(\mathrm{~s}, 1 \mathrm{H}$, naphthalene ring proton), 7.58-7.47 (m, 4H, 3 phenyl ring protons and 1 naphthalene ring proton), $7.64(\mathrm{~d}, \mathrm{~J}=8.7 \mathrm{~Hz}, 1 \mathrm{H}$, naphthalene ring proton), 7.69 (d, J=8.9 Hz, 1H, naphthalene ring proton ), 7.93 (s, 1H, naphthalene ring proton), 8.07 (d, J=6.4 Hz, 2H, phenyl ring proton). $\operatorname{MS~FAB}^{+}(\%): 381(90 \%) \mathrm{M}^{+}, 383(100 \%)[\mathrm{M}+2]^{+}$.

2-\{[(6-Bromo-2-naphthyl)oxy]methyl\}-5-(3-chlorophenyl)-1,3,4-oxadiazole (4d)

IR (KBr, $\left.\gamma_{\max } \mathrm{cm}^{-1}\right): 2975(\mathrm{C}-\mathrm{H}$ str), $1640(\mathrm{C}=\mathrm{N}), 1224(\mathrm{C}-\mathrm{O})$.

${ }^{1} \mathrm{H} \mathrm{NMR}\left(\mathrm{CDCl}_{3}, \delta \mathrm{ppm}\right): 5.46\left(\mathrm{~s}, 2 \mathrm{H}, \mathrm{O}-\mathrm{CH}_{2}\right) 7.24(\mathrm{~d}, \mathrm{~J}=6.3 \mathrm{~Hz}, 1 \mathrm{H}$, phenyl ring proton), 7.31 (s, $1 \mathrm{H}$, naphthalene ring proton), 7.37-7.47 (m, $1 \mathrm{H}$, phenyl ring protons and $2 \mathrm{H}$ naphthalene ring proton), $7.51(\mathrm{~d}, \mathrm{~J}=5.1 \mathrm{~Hz}, 1 \mathrm{H}$, phenyl ring proton), $7.62(\mathrm{~d}, \mathrm{~J}=8.8 \mathrm{~Hz}, 1 \mathrm{H}$, naphthalene ring proton), $7.67(\mathrm{~d}, \mathrm{~J}=8.9 \mathrm{~Hz}, 1 \mathrm{H}$, naphthalene ring proton), $7.93(\mathrm{~s}, 1 \mathrm{H}$, naphthalene ring proton), $7.99\left(\mathrm{~d}, \mathrm{~J}=7.6 \mathrm{~Hz}, 1 \mathrm{H}\right.$, phenyl ring proton). $\mathrm{MS} \mathrm{FAB}^{+}(\%): 415(95 \%) \mathrm{M}^{+}, 417(100 \%)[\mathrm{M}+2]^{+}$.

2-\{[(6-Bromo-2-naphthyl)oxy]methyl\}-5-(4-nitrophenyl)-1,3,4-oxadiazole (4e)

IR $\left(\mathrm{KBr}, \gamma_{\max } \mathrm{cm}^{-1}\right): 3035(\mathrm{C}-\mathrm{H}$ str), $1620(\mathrm{C}=\mathrm{N}), 1021(\mathrm{C}-\mathrm{O})$.

${ }^{1} \mathrm{H}$ NMR $\left(\mathrm{CDCl}_{3}, \delta \mathrm{ppm}\right): 5.43\left(\mathrm{~s}, 2 \mathrm{H}, \mathrm{O}-\mathrm{CH}_{2}\right), 7.24(\mathrm{~d}, \mathrm{~J}=2.4 \mathrm{~Hz}, 1 \mathrm{H}$, naphthalene ring proton), $7.30(\mathrm{~s}, 1 \mathrm{H}$, naphthalene ring proton), 7.48-7.55 (m, 2H, phenyl ring protons and $1 \mathrm{H}$ naphthalene ring proton), $7.64(\mathrm{~d}, \mathrm{~J}=8.7 \mathrm{~Hz}, 1 \mathrm{H}$, naphthalene ring proton), $7.70(\mathrm{~d}, \mathrm{~J}=8.9 \mathrm{~Hz}, 1 \mathrm{H}$, naphthalene ring proton), $7.94(\mathrm{~s}, 1 \mathrm{H}$, naphthalene ring proton), $8.26(\mathrm{~d}$, $\mathrm{J}=8.5 \mathrm{~Hz}, 2 \mathrm{H}$, phenyl ring protons). $\mathrm{MS} \mathrm{FAB}^{+}(\%): 426(90 \%) \mathrm{M}^{+}, 428(100 \%)[\mathrm{M}+2]^{+}$.

2-\{[(6-Bromo-2-naphthyl)oxy]methyl\}-5-(4-methylphenyl)-1,3,4-oxadiazole (4f)

IR (KBr, $\left.\gamma_{\max } \mathrm{cm}^{-1}\right): 2938$ (C-H str), $1645(\mathrm{C}=\mathrm{N}), 1251(\mathrm{C}-\mathrm{O})$.

${ }^{1} \mathrm{H} \mathrm{NMR}\left(\mathrm{CDCl}_{3}, \delta \mathrm{ppm}\right): 2.42$ (s, 3H, CH3), $5.43\left(\mathrm{~s}, 2 \mathrm{H}, \mathrm{O}-\mathrm{CH}_{2}\right), 7.14$ (d, J=7.6 Hz, 1H, naphthalene ring proton), $7.26(\mathrm{~d}, \mathrm{~J}=6.0 \mathrm{~Hz}, 2 \mathrm{H}$, phenyl ring protons), $7.32(\mathrm{~s}, 1 \mathrm{H}$, naphthalene ring proton), $7.39(\mathrm{~d}, \mathrm{~J}=7.1 \mathrm{~Hz}, 1 \mathrm{H}$, naphthalene ring proton), $7.53(\mathrm{~d}, \mathrm{~J}=8.7 \mathrm{~Hz}, 1 \mathrm{H}$, naphthalene ring proton), $7.65(\mathrm{~d}, \mathrm{~J}=8.8 \mathrm{~Hz}, 1 \mathrm{H}$, naphthalene ring proton), 7.69 (d, $\mathrm{J}=8.9 \mathrm{~Hz}, 1 \mathrm{H}$, naphthalene ring proton), $7.85(\mathrm{~d}, \mathrm{~J}=6.0 \mathrm{~Hz}, 2 \mathrm{H}$, phenyl ring protons), $7.97(\mathrm{~s}, 1 \mathrm{H}$, naphthalene ring proton). $\mathrm{MS} \mathrm{FAB}^{+}(\%): 395(90 \%) \mathrm{M}^{+}, 397(100 \%)[\mathrm{M}+2]^{+}$.

2-\{[(6-Bromo-2-naphthyl)oxy]methyl\}-5-(3,5-dinitrophenyl)-1,3,4-oxadiazole (4g)

IR (KBr, $\left.\gamma_{\max } \mathrm{cm}^{-1}\right): 3018$ (C-H str), $1629(\mathrm{C}=\mathrm{N}), 1092(\mathrm{C}-\mathrm{O})$.

${ }^{1} \mathrm{H}$ NMR $\left(\mathrm{CDCl}_{3}, \delta \mathrm{ppm}\right): 5.52\left(\mathrm{~s}, 2 \mathrm{H}, \mathrm{O}-\mathrm{CH}_{2}\right), 7.31-7.17(\mathrm{~m}, 2 \mathrm{H}$, naphthalene ring proton), $7.55(\mathrm{~d}, \mathrm{~J}=8.6 \mathrm{~Hz}, 1 \mathrm{H}$, naphthalene ring proton), $7.66(\mathrm{~d}, \mathrm{~J}=8.5 \mathrm{~Hz}, 1 \mathrm{H}$, naphthalene ring proton), $7.73(\mathrm{~d}, \mathrm{~J}=8.7 \mathrm{~Hz}, 1 \mathrm{H}$, naphthalene ring proton), $7.95\left(\mathrm{~s}, 1 \mathrm{H}\right.$, naphthalene ring proton), 8.21-8.25 (m, 3H, phenyl ring proton). $\mathrm{MS} \mathrm{FAB}^{+}(\%): 471(80 \%) \mathrm{M}^{+}$, $473(85 \%)[\mathrm{M}+2]^{+}$.

4-(5-\{[(6-Bromo-2-naphthyl)oxy]methyl\}-1,3,4-oxadiazol-2-yl)-2-chloropyridine (4h)

IR (KBr, $\left.\gamma_{\max } \mathrm{cm}^{-1}\right): 2968$ (C-H str), $1638(\mathrm{C}=\mathrm{N}), 1095(\mathrm{C}-\mathrm{O})$.

${ }^{1} \mathrm{H}$ NMR $\left(\mathrm{CDCl}_{3}, \delta \mathrm{ppm}\right): 5.46\left(\mathrm{~s}, 2 \mathrm{H}, \mathrm{O}-\mathrm{CH}_{2}\right), 7.23(\mathrm{~d}, \mathrm{~J}=7.2 \mathrm{~Hz}, 1 \mathrm{H}$, naphthalene ring proton), $7.31(\mathrm{~s}, 1 \mathrm{H}$, naphthalene ring proton), 7.37-7.55 (m, $2 \mathrm{H}$, pyridine ring protons and $1 \mathrm{H}$ naphthalene ring proton), $7.63(\mathrm{~d}, \mathrm{~J}=8.8 \mathrm{~Hz}$, $1 \mathrm{H}$, naphthalene ring proton), $7.68(\mathrm{~d}, \mathrm{~J}=8.8 \mathrm{~Hz}, 1 \mathrm{H}$, naphthalene ring proton), 7.91 (s, $1 \mathrm{H}$, naphthalene ring proton), $7.98\left(\mathrm{~d}, \mathrm{~J}=6.0 \mathrm{~Hz}, 1 \mathrm{H}\right.$, pyridine ring protons). $\mathrm{MS} \mathrm{FAB}^{+}(\%): 416(80 \%) \mathrm{M}^{+}, 418(85 \%)[\mathrm{M}+2]^{+}$.

2-\{[(6-Bromo-2-naphthyl)oxy]methyl\}-5-(4-chlorophenyl)-1,3,4-oxadiazole (4i)

IR (KBr, $\left.\gamma_{\max } \mathrm{cm}^{-1}\right): 2939$ (C-H str), $1642(\mathrm{C}=\mathrm{N}), 1244(\mathrm{C}-\mathrm{O})$. 
${ }^{1} \mathrm{H}$ NMR $\left(\mathrm{CDCl}_{3}, \delta \mathrm{ppm}\right): 5.43\left(\mathrm{~s}, 2 \mathrm{H}, \mathrm{O}-\mathrm{CH}_{2}\right), 7.24(\mathrm{~d}, \mathrm{~J}=2.4 \mathrm{~Hz}, 1 \mathrm{H}$, naphthalene ring proton), $7.30(\mathrm{~s}, 1 \mathrm{H}$, naphthalene ring proton), 7.55-7.48 $(\mathrm{m}, 2 \mathrm{H}$, phenyl ring protons and $1 \mathrm{H}$ naphthalene ring proton), $7.64(\mathrm{~d}, \mathrm{~J}=8.7 \mathrm{~Hz}, 1 \mathrm{H}$, naphthalene ring proton), $7.70(\mathrm{~d}, \mathrm{~J}=8.9 \mathrm{~Hz}, 1 \mathrm{H}$, naphthalene ring proton), $7.94(\mathrm{~s}, 1 \mathrm{H}$, naphthalene ring proton), $8.02(\mathrm{~d}$, $\mathrm{J}=8.5 \mathrm{~Hz}, 2 \mathrm{H}$, phenyl ring protons). $\mathrm{MS} \mathrm{FAB}^{+}(\%): 415(90 \%) \mathrm{M}^{+}, 417(100 \%)[\mathrm{M}+2]^{+}$.

2-\{[(6-Bromo-2-naphthyl)oxy]methyl\}-5-(2,4-dichlorophenyl)-1,3,4-oxadiazole (4j)

IR $\left(\mathrm{KBr}, \gamma_{\max } \mathrm{cm}^{-1}\right)$ : $2935(\mathrm{C}-\mathrm{H}$ str), $1641(\mathrm{C}=\mathrm{N}), 1090(\mathrm{C}-\mathrm{O})$.

${ }^{1} \mathrm{H}$ NMR $\left(\mathrm{CDCl}_{3}, \delta \mathrm{ppm}\right): 5.43\left(\mathrm{~s}, 2 \mathrm{H}, \mathrm{O}-\mathrm{CH}_{2}\right), 7.16(\mathrm{~d}, \mathrm{~J}=7.4 \mathrm{~Hz}, 1 \mathrm{H}$, naphthalene ring proton), 7.29 (s, 2H, naphthalene ring proton and phenyl ring proton), $7.53(\mathrm{~d}, \mathrm{~J}=8.7 \mathrm{~Hz}, 1 \mathrm{H}$, naphthalene ring proton), $7.64(\mathrm{~d}, \mathrm{~J}=8.7 \mathrm{~Hz}, 1 \mathrm{H}$, naphthalene ring proton), $7.70(\mathrm{~d}, \mathrm{~J}=8.7 \mathrm{~Hz}, 1 \mathrm{H}$, naphthalene ring proton), 7.91 (d, J=8.6 Hz, 1H, phenyl ring protons), $7.94\left(\mathrm{~s}, 1 \mathrm{H}\right.$, naphthalene ring proton), $8.06\left(\mathrm{~s}, 1 \mathrm{H}\right.$, phenyl ring proton). $\mathrm{MS} \mathrm{FAB}^{+}(\%): 450(100 \%) \mathrm{M}^{+}, 452(90 \%)$ $[\mathrm{M}+2]^{+}$.

3-(5-\{[(6-Bromo-2-naphthyl)oxy]methyl\}-1,3,4-oxadiazol-2-yl)aniline (4k)

IR (KBr, $\left.\gamma_{\max } \mathrm{cm}^{-1}\right): 2935$ (C-H str), $1629(\mathrm{C}=\mathrm{N}), 1214(\mathrm{C}-\mathrm{O})$.

${ }^{1} \mathrm{H}$ NMR $\left(\mathrm{CDCl}_{3}, \delta \mathrm{ppm}\right): 5.43$ (s, 2H, O- $\left.\mathrm{CH}_{2}\right), 6.08\left(\mathrm{~s}, 2 \mathrm{H}, \mathrm{NH}_{2}\right), 7.16$ (d, J=7.5Hz, 1H, naphthalene ring proton), 7.32 $(\mathrm{s}, 1 \mathrm{H}$, naphthalene ring protons), 7.37-7.41 ( $\mathrm{m}, 3 \mathrm{H}$, phenyl ring proton), $7.48(\mathrm{~d}, \mathrm{~J}=8.4 \mathrm{~Hz}, 1 \mathrm{H}$, phenyl ring proton), 7.53 $(\mathrm{d}, \mathrm{J}=8.7 \mathrm{~Hz}, 1 \mathrm{H}$, naphthalene ring proton), $7.64(\mathrm{~d}, \mathrm{~J}=8.7 \mathrm{~Hz}, 1 \mathrm{H}$, naphthalene ring proton), $7.70(\mathrm{~d}, \mathrm{~J}=8.9 \mathrm{~Hz}, 1 \mathrm{H}$, naphthalene ring proton), 7.92 (s, $1 \mathrm{H}$, naphthalene ring proton). $\mathrm{MS} \mathrm{FAB}^{+}(\%): 396(100 \%) \mathrm{M}^{+}, 398(95 \%)[\mathrm{M}+2]^{+}$.

2-\{[(6-Bromo-2-naphthyl)oxy]methyl\}-5-(3-methylphenyl)-1,3,4-oxadiazole (4I)

IR ( $\left.\mathrm{KBr}, \gamma_{\max } \mathrm{cm}^{-1}\right): 2964$ (C-H str), $1630(\mathrm{C}=\mathrm{N}), 1084(\mathrm{C}-\mathrm{O})$.

${ }^{1} \mathrm{H}$ NMR $\left(\mathrm{CDCl}_{3}, \delta \mathrm{ppm}\right): 2.44(\mathrm{~s}, 3 \mathrm{H}, \mathrm{CH} 3), 5.43\left(\mathrm{~s}, 2 \mathrm{H}, \mathrm{O}-\mathrm{CH}_{2}\right), 7.14(\mathrm{~d}, \mathrm{~J}=7.5 \mathrm{~Hz}, 1 \mathrm{H}$, naphthalene ring proton), $7.32(\mathrm{~s}, 1 \mathrm{H}$, naphthalene ring protons), 7.41-7.37 (m, 2H, phenyl ring proton), $7.53(\mathrm{~d}, \mathrm{~J}=8.7 \mathrm{~Hz}, 1 \mathrm{H}$, naphthalene ring proton), $7.64(\mathrm{~d}, \mathrm{~J}=8.7 \mathrm{~Hz}, 1 \mathrm{H}$, naphthalene ring proton), $7.70(\mathrm{~d}, \mathrm{~J}=8.9 \mathrm{~Hz}, 1 \mathrm{H}$, naphthalene ring proton), 7.86 (d, $\mathrm{J}=6.8$ $\mathrm{Hz}, 1 \mathrm{H}$, phenyl ring protons), $7.90\left(\mathrm{~s}, 1 \mathrm{H}\right.$, phenyl ring protons), 7.94 (s, $1 \mathrm{H}$, naphthalene ring proton). $\mathrm{MS} \mathrm{FAB}^{+}(\%)$ : $397(95 \%)[\mathrm{M}+2]^{+}, 395(100 \%) \mathrm{M}^{+}$.

2-\{[(6-Bromo-2-naphthyl)oxy]methyl\}-5-(3,5-dichlorophenyl)-1,3,4-oxadiazole (4m)

IR $\left(\mathrm{KBr}, \gamma_{\max } \mathrm{cm}^{-1}\right): 3029(\mathrm{C}-\mathrm{H}$ str), $1659(\mathrm{C}=\mathrm{N}), 1226(\mathrm{C}-\mathrm{O})$

${ }^{1} \mathrm{H}$ NMR $\left(\mathrm{CDCl}_{3}, \delta \mathrm{ppm}\right): 5.44\left(\mathrm{~s}, 2 \mathrm{H}, \mathrm{O}-\mathrm{CH}_{2}\right), 7.16(\mathrm{~d}, \mathrm{~J}=7.4 \mathrm{~Hz}, 1 \mathrm{H}$, naphthalene ring proton), $7.29(\mathrm{~s}, 1 \mathrm{H}$, naphthalene ring protons), $7.53(\mathrm{~d}, \mathrm{~J}=8.7 \mathrm{~Hz}, 1 \mathrm{H}$, naphthalene ring proton), $7.64(\mathrm{~d}, \mathrm{~J}=8.7 \mathrm{~Hz}, 1 \mathrm{H}$, naphthalene ring proton), 7.70 (d, J=8.7 Hz, 1H, naphthalene ring proton), 7.94 (s, 1H, naphthalene ring proton), 7.97 (s, 2H, phenyl ring protons), 8.02 (s, 1H, phenyl ring proton). $\mathrm{MS} \mathrm{FAB}^{+}(\%): 450(100 \%) \mathrm{M}^{+}, 452(90 \%)[\mathrm{M}+2]^{+}$.

2-\{[(6-Bromo-2-naphthyl)oxy]methyl\}-5-(1-ethoxy-2-naphthyl)-1,3,4-oxadiazole (4o)

IR ( $\left.\mathrm{KBr}, \gamma_{\max } \mathrm{cm}^{-1}\right): 2945(\mathrm{C}-\mathrm{H}$ str), $1641(\mathrm{C}=\mathrm{N}), 1234(\mathrm{C}-\mathrm{O})$.

${ }^{1} \mathrm{H}$ NMR (DMSO, $\delta$ ppm): 1.12 (t, J=6.9 Hz, 3H, $\mathrm{CH}_{3}$ ethyl group), 4.18(q, J=7.02 Hz, 2H, $\mathrm{CH}_{2}$ ethyl group), 5.68 (s, $\left.2 \mathrm{H}, \mathrm{O}-\mathrm{CH}_{2}\right), 7.31-8.22\left(\mathrm{~m}, 12 \mathrm{H}\right.$ two naphthalene ring proton). $\mathrm{MS} \mathrm{FAB}^{+}(\%): 477(90 \%)[\mathrm{M}+2]^{+}, 475(100 \%) \mathrm{M}^{+}$.

2-\{[(6-Bromo-2-naphthyl)oxy] methyl\}-5-(2-chlorophenyl)-1,3,4-oxadiazole (4p)

IR $\left(\mathrm{KBr}, \gamma_{\max } \mathrm{cm}^{-1}\right)$ : $2941(\mathrm{C}-\mathrm{H}$ str), $1638(\mathrm{C}=\mathrm{N}), 1092(\mathrm{C}-\mathrm{O})$.

${ }^{1} \mathrm{H}$ NMR $\left(\mathrm{CDCl}_{3}, \delta \mathrm{ppm}\right): 5.47\left(\mathrm{~s}, 2 \mathrm{H}, \mathrm{O}-\mathrm{CH}_{2}\right), 7.14(\mathrm{~d}, \mathrm{~J}=7.2 \mathrm{~Hz}, 1 \mathrm{H}$, naphthalene ring proton), $7.32(\mathrm{~s}, 1 \mathrm{H}$, naphthalene ring proton), 7.55-7.37 (m, 3H, phenyl ring protons and $1 \mathrm{H}$ naphthalene ring proton), $7.64(\mathrm{~d}, \mathrm{~J}=8.8 \mathrm{~Hz}, 1 \mathrm{H}$, naphthalene ring proton), $7.69(\mathrm{~d}, \mathrm{~J}=8.8 \mathrm{~Hz}, 1 \mathrm{H}$, naphthalene ring proton), $7.93(\mathrm{~s}, 1 \mathrm{H}$, naphthalene ring proton), $8.0(\mathrm{~d}$, $\mathrm{J}=6.5 \mathrm{~Hz}, 1 \mathrm{H}$, phenyl ring protons). $\mathrm{MS} \mathrm{FAB}^{+}(\%): 415(80 \%) \mathrm{M}^{+}, 417(85 \%)[\mathrm{M}+2]^{+}$.

2.1.4 General procedure for the preparation of 5-\{[(6-bromo-2-naphthyl)oxy]methyl $\}-1,3,4$-oxadiazole-2(3H)-thione (5)

2-[(6-Bromo-2-naphthyl)oxy]acetohydrazide ( $10.0 \mathrm{~g}, 0.033 \mathrm{~mole})$ was treated with a solution of potassium hydroxide $(2.8 \mathrm{~g}, 0.05 \mathrm{~mole})$ dissolved in methanol $(100 \mathrm{ml})$ under stirring. Carbon disulfide $(3.8 \mathrm{~g}, 0.05$ mole) was added slowly to the reaction mixture. The reaction mixture was slowly heated to reflux and refluxed for $8 \mathrm{~h}$. The solvent was distilled under vacuum and the residue was dissolved in water. Acidified the solution and collected the resulting solid by filtration. Yield $86 \%$. m.p $148-150{ }^{\circ} \mathrm{C}$.

Elemental analysis \% Calculated (found) for $\mathrm{C}_{13} \mathrm{H}_{9} \mathrm{BrN}_{2} \mathrm{O}_{2} \mathrm{~S} \quad \mathrm{C}-46.30(46.31), \mathrm{H}-2.69(2.67)$ and N- 8.30(8.29).

IR (KBr) cm ${ }^{-1}: 3400(\mathrm{NH}), 2950(\mathrm{CH}), 1255(\mathrm{C}=\mathrm{S}), 1635(\mathrm{C}=\mathrm{N}$ cyclic). 
${ }^{1} \mathrm{H}$ NMR (DMSO-d $\mathrm{d}_{6}, \delta \mathrm{ppm}$ ): 5.37 (s, 2H, O-CH ${ }_{2}$ ), 7.29 (d, J=7.1 Hz, 1H, naphthalene ring proton), 7.42 (d, J=7.1 Hz, $1 \mathrm{H}$, naphthalene ring proton), $7.52(\mathrm{~s}, 1 \mathrm{H}$, naphthalene ring proton), $7.78(\mathrm{~d}, \mathrm{~J}=8.85 \mathrm{~Hz}, 1 \mathrm{H}$, naphthalene ring proton), $7.87(\mathrm{~d}, \mathrm{~J}=8.8 \mathrm{~Hz}, 1 \mathrm{H}$, naphthalene ring proton), 8.15 (s, 1H, naphthalene ring proton), 15.1 (bs, 1H, SH).

FAB MS: 337(100) $\mathrm{M}^{+}, 339(40)[\mathrm{M}+2]^{+}$.

\subsubsection{Procedure for the preparation of Mannich bases (6a-j)}

To a mixture of 5-\{[(6-bromo-2-naphthyl)oxy]methyl $\}$-1,3,4-oxadiazole-2(3H)-thione(5) (1.0 g, $3.0 \mathrm{mmol})$ in methanol $(5 \mathrm{ml})$ was added formaldehyde $(0.5 \mathrm{ml}, 37 \%)$ and appropriate primary or secondary amine $(3.0 \mathrm{mmol})$. The reaction mixture was stirred overnight. After cooling, the precipitate was filtered and crystallized from methanol. The characterization data are given in Table 2.

5-\{[(6-Bromo-2-naphthyl)oxy]methyl\}-3-(morpholin-4-ylmethyl)-1,3,4-oxadiazole-2(3H)-thione (6a)

IR $\left(\mathrm{KBr}, \gamma_{\max } \mathrm{cm}^{-1}\right): 3035(\mathrm{C}-\mathrm{H}$ str), $1651(\mathrm{C}=\mathrm{N}), 1325(\mathrm{C}=\mathrm{S}), 1236(\mathrm{C}-\mathrm{O})$.

${ }^{1} \mathrm{H} \mathrm{NMR}\left(\mathrm{CDCl}_{3}, \delta \mathrm{ppm}\right): 2.77$ (t, J=4.6 Hz, 4H, morpholine protons), 3.67 (t, J=4.4 Hz, 4H, morpholine protons), $4.99\left(\mathrm{~s}, 2 \mathrm{H}, \mathrm{O}-\mathrm{CH}_{2}\right), 5.15\left(\mathrm{~s}, 2 \mathrm{H}, \mathrm{N}-\mathrm{CH}_{2}\right), 7.20(\mathrm{~d}, \mathrm{~J}=7.4 \mathrm{~Hz}, 1 \mathrm{H}$, naphthalene ring proton), $7.23(\mathrm{~s}, 1 \mathrm{H}$, naphthalene ring proton) $7.54(\mathrm{~d}, \mathrm{~J}=8.7 \mathrm{~Hz}, 1 \mathrm{H}$, naphthalene ring proton), $7.62(\mathrm{~d}, \mathrm{~J}=8.7 \mathrm{~Hz}, 1 \mathrm{H}$, naphthalene ring proton), $7.71(\mathrm{~d}, \mathrm{~J}=9.0$ $\mathrm{Hz}, 1 \mathrm{H}$, naphthalene ring proton), $7.95(\mathrm{~s}, 1 \mathrm{H}$, naphthalene ring proton).

FAB MS: 436(100) $\mathrm{M}^{+}, 438(85)[\mathrm{M}+2]^{+}$.

5-\{[(6-Bromo-2-naphthyl)oxy]methyl\}-3-[(4-methylpiperazin-1-yl)methyl]-1,3,4-oxadiazole-2(3H)-thione (6b)

IR ( $\left.\mathrm{KBr}, \gamma_{\max } \mathrm{cm}^{-1}\right): 2935$ (C-H str), $1650(\mathrm{C}=\mathrm{N}), 1320(\mathrm{C}=\mathrm{S}), 1224(\mathrm{C}-\mathrm{O})$.

${ }^{1} \mathrm{H} \mathrm{NMR}\left(\mathrm{CDCl}_{3}, \delta \mathrm{ppm}\right): 2.25$ (s, 3H, $\mathrm{CH}_{3}$ of piperazine), $2.40\left(\mathrm{t}, \mathrm{J}=7.2 \mathrm{~Hz}, 4 \mathrm{H}, \mathrm{CH}_{2}\right.$ of piperzaine), $2.42(\mathrm{t}, \mathrm{J}=7.2 \mathrm{~Hz}$, $4 \mathrm{H}, \mathrm{CH}_{2}$ piperzaine), 4.97(s, $\left.2 \mathrm{H}, \mathrm{O}-\mathrm{CH}_{2}\right), 5.13\left(\mathrm{~s}, 2 \mathrm{H}, \mathrm{N}-\mathrm{CH}_{2}\right), 7.09(\mathrm{~d}, \mathrm{~J}=7.4 \mathrm{~Hz}, 1 \mathrm{H}$, naphthalene ring proton), $7.21(\mathrm{~s}$, $1 \mathrm{H}$, naphthalene ring proton), $7.53(\mathrm{~d}, \mathrm{~J}=8.7 \mathrm{~Hz}, 1 \mathrm{H}$ naphthalene ring proton), $7.61(\mathrm{~d}, \mathrm{~J}=8.7 \mathrm{~Hz}, 1 \mathrm{H}$, naphthalene ring proton), $7.73(\mathrm{~d}, \mathrm{~J}=9.0 \mathrm{~Hz}, 1 \mathrm{H}$, naphthalene ring proton), $7.96(\mathrm{~s}, 1 \mathrm{H}$, naphthalene ring proton).

FAB MS: 449(95) $\mathrm{M}^{+}, 451(20)[\mathrm{M}+2]^{+}$.

5-\{[(6-Bromo-2-naphthyl)oxy]methyl\}-3-(piperidin-1-ylmethyl)-1,3,4-oxadiazole-2(3H)-thione (6c)

IR $\left(\mathrm{KBr}, \gamma_{\max } \mathrm{cm}^{-1}\right): 3015(\mathrm{C}-\mathrm{H}$ str), $1624(\mathrm{C}=\mathrm{N}), 1298(\mathrm{C}=\mathrm{S}), 1086(\mathrm{C}-\mathrm{O})$.

${ }^{1} \mathrm{H}$ NMR $\left(\mathrm{CDCl}_{3}, \delta \mathrm{ppm}\right):$ 2.10-2.63 (m, 6H, piperidine protons), $2.78\left(\mathrm{t}, \mathrm{J}=5.2 \mathrm{~Hz}, 4 \mathrm{H}, \mathrm{CH}_{2}-\mathrm{N}-\mathrm{CH}_{2}\right.$ piperidine protons), $4.99\left(\mathrm{~s}, 2 \mathrm{H}, \mathrm{O}-\mathrm{CH}_{2}\right), 5.15\left(\mathrm{~s}, 2 \mathrm{H}, \mathrm{N}-\mathrm{CH}_{2}\right), 7.18(\mathrm{~d}, \mathrm{~J}=7.4 \mathrm{~Hz}, 1 \mathrm{H}$, naphthalene ring proton), $7.23(\mathrm{~s}, 1 \mathrm{H}$, naphthalene ring proton), $7.54(\mathrm{~d}, \mathrm{~J}=8.7 \mathrm{~Hz}, 1 \mathrm{H}$ naphthalene ring proton), $7.62(\mathrm{~d}, \mathrm{~J}=8.7 \mathrm{~Hz}, 1 \mathrm{H}$, naphthalene ring proton), $7.71(\mathrm{~d}, \mathrm{~J}=9.0 \mathrm{~Hz}, 1 \mathrm{H}$, naphthalene ring proton), $7.95(\mathrm{~s}, 1 \mathrm{H}$, naphthalene ring proton).

FAB MS: $434(100) \mathrm{M}^{+}, 436(60)[\mathrm{M}+2]^{+}$

5-\{[(6-Bromo-2-naphthyl)oxy]methyl\}-3-[(diethylamino)methyl]-1,3,4-oxadiazole-2(3H)-thione (6d)

IR $\left(\mathrm{KBr}, \gamma_{\max } \mathrm{cm}^{-1}\right): 3035(\mathrm{C}-\mathrm{H}$ str), $1650(\mathrm{C}=\mathrm{N}), 1324(\mathrm{C}=\mathrm{S}), 1216(\mathrm{C}-\mathrm{O})$.

${ }^{1} \mathrm{H}$ NMR $\left(\mathrm{CDCl}_{3}, \delta \mathrm{ppm}\right): 1.07$ (t, 6H, Two $\mathrm{CH}_{3}$ protons), 2.97 (q, 4H, $\mathrm{CH}_{2}-\mathrm{N}-\mathrm{CH}_{2}$ protons), 4.99 (s, 2H, O-CH $), 5.15$ (s, $\left.2 \mathrm{H}, \mathrm{N}-\mathrm{CH}_{2}\right), 7.17(\mathrm{~d}, \mathrm{~J}=7.4 \mathrm{~Hz}, 1 \mathrm{H}$, naphthalene ring proton), $7.23(\mathrm{~s}, 1 \mathrm{H}$, naphthalene ring proton), 7.54 (d, $\mathrm{J}=8.7$ $\mathrm{Hz}, 1 \mathrm{H}$, naphthalene ring proton ), $7.62(\mathrm{~d}, \mathrm{~J}=8.7 \mathrm{~Hz}, 1 \mathrm{H}$, naphthalene ring proton), 7.71(d, $\mathrm{J}=9.0 \mathrm{~Hz}, 1 \mathrm{H}$, naphthalene ring proton), $7.95(\mathrm{~s}, 1 \mathrm{H}$, naphthalene ring proton).

FAB MS: $422(95) \mathrm{M}^{+}, 424(65)[\mathrm{M}+2]^{+}$.

5-\{[(6-Bromo-2-naphthyl)oxy]methyl\}-3-(1H-imidazol-1-ylmethyl)-1,3,4-oxadiazole-2(3H)-thione (6e)

IR ( $\left.\mathrm{KBr}, \gamma_{\max } \mathrm{cm}^{-1}\right): 3033$ (C-H str), $1648(\mathrm{C}=\mathrm{N}), 1318(\mathrm{C}=\mathrm{S}), 1235(\mathrm{C}-\mathrm{O})$.

${ }^{1} \mathrm{H}$ NMR $\left(\mathrm{CDCl}_{3}, \delta \mathrm{ppm}\right): 5.02\left(\mathrm{~s}, 2 \mathrm{H}, \mathrm{O}-\mathrm{CH}_{2}\right), 5.47\left(\mathrm{~s}, 2 \mathrm{H}, \mathrm{N}-\mathrm{CH}_{2}\right), 7.16(\mathrm{~d}, \mathrm{~J}=7.4 \mathrm{~Hz}, 1 \mathrm{H}$, naphthalene ring proton), $7.23(\mathrm{~m}, 1 \mathrm{H}$, naphthalene ring proton and $2 \mathrm{H}$ imidazole ring protons) $7.54(\mathrm{~d}, \mathrm{~J}=8.7 \mathrm{~Hz}, 1 \mathrm{H}$, naphthalene ring proton), $7.62(\mathrm{~d}, \mathrm{~J}=8.7 \mathrm{~Hz}, 1 \mathrm{H}$, naphthalene ring proton), $7.71(\mathrm{~d}, \mathrm{~J}=9.0 \mathrm{~Hz}, 1 \mathrm{H}$, naphthalene ring proton), 7.81 (s, $1 \mathrm{H}$, imidazole ring proton), 7.95 (s, $1 \mathrm{H}$, naphthalene ring proton).

FAB MS: $417(80) \mathrm{M}^{+}, 419(30)[\mathrm{M}+2]^{+}$.

5-\{[(6-Bromo-2-naphthyl)oxy] methyl\}-3-\{[(2-fluorophenyl)amino]methyl\}-1,3,4-oxadiazole-2(3H)-thione (6f)

IR (KBr, $\left.\gamma_{\max } \mathrm{cm}^{-1}\right): 3031(\mathrm{C}-\mathrm{H}$ str), $2966(\mathrm{NH}), 1624(\mathrm{C}=\mathrm{N}), 1325(\mathrm{C}=\mathrm{S}), 1208(\mathrm{C}-\mathrm{O})$.

${ }^{1} \mathrm{H}$ NMR ( $\left.\mathrm{CDCl}_{3}, \delta \mathrm{ppm}\right): 5.37$ (s, 2H, O-CH $), 5.45$ (d, J=7.0 Hz, 2H, N-CH $\left.{ }_{2}-\mathrm{NH}\right), 6.97$ (bs, NH proton), 7.23-7.33 (m, $3 \mathrm{H}$, phenyl ring proton and $2 \mathrm{H}$, naphthalene ring proton), $7.54(\mathrm{~d}, \mathrm{~J}=8.3 \mathrm{~Hz}, 1 \mathrm{H}$, naphthalene ring proton), $7.65(\mathrm{~d}$, 
$\mathrm{J}=8.6 \mathrm{~Hz}, 1 \mathrm{H}$, naphthalene ring proton), $7.70(\mathrm{~d}, \mathrm{~J}=8.8 \mathrm{~Hz}, 1 \mathrm{H}$, naphthalene ring proton), 7.94 (s, 1H, naphthalene ring proton), 8.0 ( $\mathrm{t}, \mathrm{J}=7.8 \mathrm{~Hz}, 1 \mathrm{H}$, phenyl ring protons).

FAB MS: 460(80) $\mathrm{M}^{+}, 462(20)[\mathrm{M}+2]^{+}$.

5-\{[(6-Bromo-2-naphthyl)oxy]methyl\}-3-\{[(4-chlorophenyl)amino]methyl $\}-1,3,4$-oxadiazole-2(3H)-thione (6g)

IR $\left(\mathrm{KBr}, \gamma_{\max } \mathrm{cm}^{-1}\right): 3072(\mathrm{C}-\mathrm{H}$ str), $2978(\mathrm{NH}), 1640(\mathrm{C}=\mathrm{N}), 1326(\mathrm{C}=\mathrm{S}), 1088(\mathrm{C}-\mathrm{O})$.

${ }^{1} \mathrm{H}$ NMR $\left(\mathrm{CDCl}_{3}, \delta\right.$ ppm): 5.43 (s, 2H, O-CH $), 5.55$ (d, J=7.0 Hz, 2H, N-CH $\left.{ }_{2}-\mathrm{NH}\right), 6.96$ (bs, NH proton), 7.24 (d, $\mathrm{J}=8.4 \mathrm{~Hz}, 1 \mathrm{H}$, naphthalene ring proton), $7.30(\mathrm{~s}, 1 \mathrm{H}$, naphthalene ring proton), 7.55-7.48 (m, $2 \mathrm{H}$, phenyl ring protons and $1 \mathrm{H}$ naphthalene ring proton), $7.64(\mathrm{~d}, \mathrm{~J}=8.7 \mathrm{~Hz}, 1 \mathrm{H}$ naphthalene ring proton), $7.70(\mathrm{~d}, \mathrm{~J}=8.9 \mathrm{~Hz}, 1 \mathrm{H}$, naphthalene ring proton), 7.94 (s, $1 \mathrm{H}$, naphthalene ring proton), 8.02 (d, J=8.5 Hz, 2H, phenyl ring protons).

FAB MS: 476(85) $\mathrm{M}^{+}, 478(60)[\mathrm{M}+2]^{+}$.

5-\{[(6-Bromo-2-naphthyl)oxy]methyl\}-3-\{[(2-methylphenyl)amino $]$ methyl\}-1,3,4-oxadiazole-2(3H)-thione (6h)

IR $\left(\mathrm{KBr}, \gamma_{\max } \mathrm{cm}^{-1}\right)$ : $3031(\mathrm{C}-\mathrm{H}$ str), $2986(\mathrm{NH}), 1628(\mathrm{C}=\mathrm{N}), 1319(\mathrm{C}=\mathrm{S}), 1067(\mathrm{C}-\mathrm{O})$.

${ }^{1} \mathrm{H}$ NMR $\left(\mathrm{CDCl}_{3}, \delta \mathrm{ppm}\right): 2.28$ (s, 3H, $\left.-\mathrm{CH}_{3}\right), 5.07\left(\mathrm{~s}, 2 \mathrm{H}, \mathrm{O}-\mathrm{CH}_{2}\right), 5.45$ (d, J=7.0 Hz, 2H, N-CH $\left.2-\mathrm{NH}\right), 7.12$ (bs, NH proton), $7.15(\mathrm{~s}, 1 \mathrm{H}$, naphthalene ring proton), 7.33-7.21 (m, $4 \mathrm{H}$ phenyl ring protons and $1 \mathrm{H}$ naphthalene ring proton), $7.46(\mathrm{~d}, \mathrm{~J}=8.7 \mathrm{~Hz}, 1 \mathrm{H}$, naphthalene ring proton), $7.55(\mathrm{~d}, \mathrm{~J}=8.7 \mathrm{~Hz}, 1 \mathrm{H}$, naphthalene ring proton), $7.64(\mathrm{~d}, \mathrm{~J}=9.0 \mathrm{~Hz}, 1 \mathrm{H}$ naphthalene ring proton), $7.89(\mathrm{~s}, 1 \mathrm{H}$, naphthalene ring proton).

FAB MS: $456(90) \mathrm{M}^{+}, 458(65)[\mathrm{M}+2]^{+}$.

5-\{[(6-Bromo-2-naphthyl)oxy]methyl\}-3-\{[(3-methoxyphenyl)amino]methyl\}-1,3,4-oxadiazole-2(3H)-thione (6j)

IR $\left(\mathrm{KBr}, \gamma_{\max } \mathrm{cm}^{-1}\right): 3021(\mathrm{C}-\mathrm{H}$ str), $2849(\mathrm{NH}), 1640(\mathrm{C}=\mathrm{N}), 1327(\mathrm{C}=\mathrm{S}), 1092(\mathrm{C}-\mathrm{O})$.

${ }^{1} \mathrm{H}$ NMR $\left(\mathrm{CDCl}_{3}, \delta\right.$ ppm): 3.93 (s, 3H, $\mathrm{OCH}_{3}$ ), 5.06 (s, 2H, O-CH $), 5.42\left(\mathrm{~d}, \mathrm{~J}=6.9 \mathrm{~Hz}, 2 \mathrm{H}, \mathrm{N}-\mathrm{CH}_{2}-\mathrm{NH}\right), 7.10$ (bs, NH proton), $7.15(\mathrm{~s}, 1 \mathrm{H}$, naphthalene ring proton), 7.33-7.21 ( $\mathrm{m}, 4 \mathrm{H}$ phenyl ring protons and $1 \mathrm{H}$ naphthalene ring proton), $7.46(\mathrm{~d}, \mathrm{~J}=8.7 \mathrm{~Hz}, 1 \mathrm{H}$, naphthalene ring proton $), 7.55(\mathrm{~d}, \mathrm{~J}=8.7 \mathrm{~Hz}, 1 \mathrm{H}$, naphthalene ring proton), $7.64(\mathrm{~d}, \mathrm{~J}=8.9 \mathrm{~Hz}, 1 \mathrm{H}$, naphthalene ring proton), 7.89 (s, 1H, naphthalene ring proton).

FAB MS: $472(85) \mathrm{M}^{+}, 474(35)[\mathrm{M}+2]^{+}$.

2.1.6 Procedure for the preparation of 2-\{[(6-bromo-2-naphthyl)oxy $]$ methyl $\}-5$-(methyl/ethyl thio)-1,3,4-oxadiazole (7a-b)

5-\{[(6-Bromo-2-naphthyl)oxy]methyl $\}$-1,3,4-oxadiazole-2(3H)-thione (5) (2.0 g, $5.9 \mathrm{mmol})$ was dissolved in ethanol (5 $\mathrm{ml})$ and $10 \%$ aqueous sodium hydroxide $(3.5 \mathrm{ml})$. Methyl/ethyl iodide $(6.2 \mathrm{mmol})$ was added and sonicated for $20 \mathrm{~min}$. Water was added and the solid obtained was filtered and then crystallized from methanol.

The characterization data are given in Table 3.

2-\{[(6-Bromo-2-naphthyl)oxy]methyl\}-5-(methylthio)-1,3,4-oxadiazole (7a)

IR (KBr, $\left.\gamma_{\max } \mathrm{cm}^{-1}\right): 2938$ (C-H str), $1651(\mathrm{C}=\mathrm{N}), 1238(\mathrm{C}-\mathrm{O})$.

${ }^{1} \mathrm{H}$ NMR $\left(\mathrm{CDCl}_{3}, \delta \mathrm{ppm}\right): 2.73\left(\mathrm{~s}, 3 \mathrm{H}, \mathrm{SCH}_{3}\right), 5.33\left(\mathrm{~s}, 2 \mathrm{H}, \mathrm{O}-\mathrm{CH}_{2}\right), 7.24-7.20(\mathrm{~m}, 2 \mathrm{H}$, naphthalene ring proton), $7.52(\mathrm{~d}$, $\mathrm{J}=8.7 \mathrm{~Hz}, 1 \mathrm{H}$, naphthalene ring proton $), 7.62(\mathrm{~d}, \mathrm{~J}=8.7 \mathrm{~Hz}, 1 \mathrm{H}$ naphthalene ring proton), $7.67(\mathrm{~d}, \mathrm{~J}=8.9 \mathrm{~Hz}, 1 \mathrm{H}$, naphthalene ring proton), 7.93 ( $\mathrm{s}, 1 \mathrm{H}$, naphthalene ring proton).

FAB MS: 351(100) $\mathrm{M}^{+}, 353(30)[\mathrm{M}+2]^{+}$.

2-\{[(6-Bromo-2-naphthyl)oxy]methyl\}-5-(ethylthio)-1,3,4-oxadiazole (7b)

IR ( $\left.\mathrm{KBr}, \gamma_{\max } \mathrm{cm}^{-1}\right): 2965$ (C-H str), $1640(\mathrm{C}=\mathrm{N}), 1254(\mathrm{C}-\mathrm{O})$.

${ }^{1} \mathrm{H} \mathrm{NMR}\left(\mathrm{CDCl}_{3}, \delta \mathrm{ppm}\right): 1.56\left(\mathrm{t}, \mathrm{J}=7.2 \mathrm{~Hz}, 3 \mathrm{H}, \mathrm{CH}_{3}\right), 3.30\left(\mathrm{q}, \mathrm{J}=7.2 \mathrm{~Hz}, 2 \mathrm{H}, \mathrm{CH}_{2}\right), 5.34\left(\mathrm{~s}, 2 \mathrm{H}, \mathrm{O}_{-} \mathrm{CH}_{2}\right), 7.24-7.27(\mathrm{~m}$, $2 \mathrm{H}$, naphthalene ring proton), $7.56(\mathrm{~d}, \mathrm{~J}=8.7 \mathrm{~Hz}, 1 \mathrm{H}$, naphthalene ring proton ), $7.64(\mathrm{~d}, \mathrm{~J}=8.6 \mathrm{~Hz}, 1 \mathrm{H}$, naphthalene ring proton), 7.70 (d, J=8.89 Hz, 1H, naphthalene ring proton), 7.95 (s, 1H, naphthalene ring proton)

FAB MS: $365(100) \mathrm{M}^{+}, 367(60)[\mathrm{M}+2]^{+}$.

2.1.7 Procedure for the preparation of 2-\{[(6-bromo-2-naphthyl)oxy]methyl $\}-5-[(\operatorname{aryl})$ thio]-1,3,4-oxadiazole (7c-f)

5-\{[(6-Bromo-2-naphthyl)oxy]methyl $\}-1,3,4$-oxadiazole-2(3H)-thione (5) $(1.0 \mathrm{~g}, 3.0 \mathrm{mmol})$ in $10 \mathrm{ml} \mathrm{DMF}$ was refluxed for $5 \mathrm{~h}$ with substituted benzyl chloride $(3.0 \mathrm{mmol})$ in presence of $0.5 \mathrm{~g}$ potassium carbonate. After completion of reaction, the mixture was filtered and concentrated the filtrate. The residue was quenched to water. The solid obtained was collected by filtration and recrystallized in methanol

The characterization data are given in Table 3.

2-\{[(6-Bromo-2-naphthyl)oxy]methyl\}-5-[(2,4-dichlorobenzyl)thio]-1,3,4-oxadiazole (7c) 
IR $\left(\mathrm{KBr}, \gamma_{\max } \mathrm{cm}^{-1}\right): 3025(\mathrm{C}-\mathrm{H}$ str), $1594(\mathrm{C}=\mathrm{N}), 1184(\mathrm{C}-\mathrm{O})$.

${ }^{1} \mathrm{H} \mathrm{NMR}\left(\mathrm{CDCl}_{3}, \delta \mathrm{ppm}\right): 4.51\left(\mathrm{~s}, 2 \mathrm{H}, \mathrm{SCH}_{2}\right), 5.30\left(\mathrm{~s}, 2 \mathrm{H}, \mathrm{O}-\mathrm{CH}_{2}\right), 7.13(\mathrm{~d}, \mathrm{~J}=9.0 \mathrm{~Hz}, 1 \mathrm{H}$, naphthalene ring proton ), $7.18(\mathrm{~s}, 1 \mathrm{H}$ naphthalene ring proton), $7.23(\mathrm{~d}, \mathrm{~J}=9.0 \mathrm{~Hz}, 1 \mathrm{H}$, naphthalene ring proton), 7.39 (s, $1 \mathrm{H}$, phenyl ring proton), $7.45(\mathrm{~d}, \mathrm{~J}=6.0 \mathrm{~Hz}, 1 \mathrm{H}$, phenyl ring proton), $7.52(\mathrm{~d}, \mathrm{~J}=6.0 \mathrm{~Hz}, 1 \mathrm{H}$, phenyl ring proton), $7.6(\mathrm{~d}, \mathrm{~J}=9.0 \mathrm{~Hz}, 1 \mathrm{H}$, naphthalene ring proton), $7.67(\mathrm{~d}, \mathrm{~J}=9.0 \mathrm{~Hz}, 1 \mathrm{H}$, naphthalene ring proton), $7.92(\mathrm{~s}, 1 \mathrm{H}$, naphthalene ring proton)

FAB MS: $496(100) \mathrm{M}^{+}, 498(30)[\mathrm{M}+2]^{+}$.

2-\{[(6-Bromo-2-naphthyl)oxy]methyl\}-5-[(2-chlorobenzyl)thio]-1,3,4-oxadiazole (7d)

IR (KBr, $\left.\gamma_{\max } \mathrm{cm}^{-1}\right): 2969$ (C-H str), $1621(\mathrm{C}=\mathrm{N}), 1214(\mathrm{C}-\mathrm{O})$.

${ }^{1} \mathrm{H}$ NMR $\left(\mathrm{CDCl}_{3}, \delta \mathrm{ppm}\right): 4.58\left(\mathrm{~s}, 2 \mathrm{H}, \mathrm{S}-\mathrm{CH}_{2}\right), 5.32\left(\mathrm{~s}, 2 \mathrm{H}, \mathrm{O}-\mathrm{CH}_{2}\right), 7.17$ (s, 1H naphthalene ring proton), 7.26-7.19(m, $2 \mathrm{H}$, phenyl ring proton, $1 \mathrm{H}$ naphthalene ring proton), $7.38(\mathrm{~d}, \mathrm{~J}=6.0 \mathrm{~Hz}, 1 \mathrm{H}$, phenyl ring proton $), 7.53(\mathrm{~d}, \mathrm{~J}=9.0 \mathrm{~Hz}, 1 \mathrm{H}$, naphthalene ring proton ), $7.55(\mathrm{~d}, \mathrm{~J}=6.2 \mathrm{~Hz}, 1 \mathrm{H}$, phenyl ring proton), $7.63(\mathrm{~d}, \mathrm{~J}=9.0 \mathrm{~Hz}, 1 \mathrm{H}$, naphthalene ring proton), $7.67(\mathrm{~d}, \mathrm{~J}=9.0 \mathrm{~Hz}, 1 \mathrm{H}$, naphthalene ring proton), $7.93(\mathrm{~s}, 1 \mathrm{H}$, naphthalene ring proton)

FAB MS: 461(95) $\mathrm{M}^{+}, 463(20)[\mathrm{M}+2]^{+}$.

2-\{[(6-Bromo-2-naphthyl)oxy]methyl\}-5-[(2-methylbenzyl)thio]-1,3,4-oxadiazole (7f)

IR (KBr, $\left.\gamma_{\max } \mathrm{cm}^{-1}\right): 2939$ (C-H str), $1642(\mathrm{C}=\mathrm{N}), 1218(\mathrm{C}-\mathrm{O})$.

${ }^{1} \mathrm{H} \mathrm{NMR}\left(\mathrm{CDCl}_{3}, \delta \mathrm{ppm}\right): 2.31\left(\mathrm{~s}, 3 \mathrm{H}, \mathrm{CH}_{3}\right), 4.43\left(\mathrm{~s}, 2 \mathrm{H}, \mathrm{SCH}_{2}\right), 5.30\left(\mathrm{~s}, 2 \mathrm{H}, \mathrm{O}-\mathrm{CH}_{2}\right), 7.1(\mathrm{~s}, 1 \mathrm{H}$, naphthalene ring proton ), 7.25-7.15 (m, $4 \mathrm{H}$, phenyl ring proton and $1 \mathrm{H}$ naphthalene ring proton), $7.51(\mathrm{~d}, \mathrm{~J}=9.0 \mathrm{~Hz}, 1 \mathrm{H}$, naphthalene ring proton), $7.60(\mathrm{~d}, \mathrm{~J}=9.0 \mathrm{~Hz}, 1 \mathrm{H}$, naphthalene ring proton), $7.65(\mathrm{~d}, \mathrm{~J}=9.0 \mathrm{~Hz}, 1 \mathrm{H}$ naphthalene ring proton ), $7.91(\mathrm{~s}$, $1 \mathrm{H}$, naphthalene ring proton). FAB MS:441(100) $\mathrm{M}^{+}, 443(60)[\mathrm{M}+2]^{+}$.

\subsection{Pharmacology}

2.2.1 Antibacterial studies: The newly synthesized compounds were screened for their antibacterial activity against Escherichia coli (ATTC-25922), Staphylococcus aureus (ATTC-25923), Pseudomonas aeruginosa (ATCC-27853) and Klebsiella pneumoniae (recultured) bacterial strains by serial plate dilution method (Barry A L 1991, James D 1970). Serial dilutions of the drug in Mueller-Hinton broth were taken in tubes and their $\mathrm{pH}$ was adjusted to 5.0 using phosphate buffer. A standardized suspension of the test bacterium was inoculated and incubated for $16-18 \mathrm{~h}$ at $37^{\circ} \mathrm{C}$. The minimum inhibitory concentration (MIC) was noted by seeing the lowest concentration of the drug at which there was no visible growth.

A number of antimicrobial discs were placed on the agar for the sole purpose of producing zones of inhibition in the bacterial lawn. Twenty milliliters of agar media was poured into each Petri dish. Excess of suspension was decanted and plates were dried by placing in an incubator at $37^{\circ} \mathrm{C}$ for an hour. Using a punch, wells were made on these seeded agar plates and minimum inhibitory concentrations of the test compounds in dimethylsulfoxide (DMSO) were added into each labeled well. A control was also prepared for the plates in the same way using solvent DMSO. The Petri dishes were prepared in triplicate and maintained at $37^{\circ} \mathrm{C}$ for 3-4 days. Antibacterial activity was determined by measuring the diameter of inhibition zone. Activity of each compound was compared with Ciprofloxacin as standard (Felon C H 1986). Zone of inhibition was determined for newly synthesized compounds and the results are summarized in Table 4.

2.2.2 Antifungal studies: Newly prepared compounds were screened for their antifungal activity against Aspergilus flavus (NCIM No.524), Aspergilus fumigates (NCIM No. 902), Penicillium (S.aurus) (recultured) and Trichophyton mentagrophytes (recultured) in DMSO by serial plate dilution method (Arthington-skaggs 2000, Verma R S. 1998). Sabourands agar media was prepared by dissolving peptone (1 g), D-glucose (4 g) and agar (2 g) in distilled water (100 $\mathrm{ml}$ ) and adjusting the $\mathrm{pH}$ to 5.7. Normal saline was used to make a suspension of spore of fungal strains for lawning. A loopful of particular fungal strain was transferred to $3 \mathrm{ml}$ saline to get a suspension of corresponding species. Twenty milliliters of agar media was poured into each Petri dish. Excess of suspension was decanted and plates were dried by placing in incubator at $37^{\circ} \mathrm{C}$ for $1 \mathrm{~h}$. Using a punch, wells were made on these seeded agar plates minimum inhibitory concentrations of the test compounds in DMSO were added into each labeled well. A control was also prepared for the plates in the same way using solvent DMSO. The Petri dishes were prepared in triplicate and maintained at $37{ }^{\circ} \mathrm{C}$ for 3-4 days. Antifungal activity was determined by measuring the diameter of inhibition zone. Activity of each compound was compared with Cyclopiroxolamine as standard. Zones of inhibition were determined and the results are summarized in Table 5.

\section{Result and discussion}

\subsection{Chemistry}

1,3,4-Oxadiazoles were generally prepared by treating carboxylic acid and acid hydrazide through cyclization in presence of chlorinating agent, which performs dehydration. Generally $\mathrm{POCl}_{3}$ is used as chlorinating reagent, which either used as such or in combination with a solvent. The reaction sequences employed for synthesis of title compound 
is shown in scheme-1. The key intermediate, ethyl (6-bromo-2-naphthyloxy)acetate (2) was prepared by treating ethyl chloroacetate with 6-bromonaphthol (1) by boiling in dry acetone in presence of potassium carbonate. The ester (2) was conveniently converted to 2-[(6-bromo-2-naphthyl)oxy]acetohydrazide (3) by refluxing with hydrazine hydrate in ethanol. Condensation of (3) with various aromatic carboxylic acids in presence of boiling phosphorous oxychloride yielded 2-\{[(6-bromo-2-naphthyl)oxy]methyl $\}-5$-aryl-1,3,4-oxadiazole (4a-r) in moderate to good yield. The structure of newly synthesized compounds were established on the basis of elemental analysis and spectral (IR, ${ }^{1} \mathrm{H}$ NMR and FAB mass) data.

The IR spectrum of 2-[(6-bromo-2-naphthyl)oxy]acetohydrazide (3) showed absorption band in the region of $3311 \mathrm{~cm}^{-1}$ characteristic of $\mathrm{NH}_{2}$ group and at $3206 \mathrm{~cm}^{-1}$ of $\mathrm{NH}$ group. The $\mathrm{C}=\mathrm{O}$ stretching was observed at $1667 \mathrm{~cm}^{-1}$. The ${ }^{1} \mathrm{H}$ NMR spectrum showed a broad singlet at $\delta 4.35$ integrating for two protons were due to $\mathrm{NH}_{2}$ group. The O- $\mathrm{CH}_{2}$ - proton came into resonance at $\delta 4.60$ as singlet. The 5 protons of naphthalene ring resonated at $\delta 7.28-7.75$. The $\mathrm{C}_{5}-\mathrm{H}$ proton appeared as singlet at $\delta 7.84$ and a broad singlet at $\delta 9.41$ for $\mathrm{NH}$ proton. The mass spectrum of this compound showed molecular ion peak at $\mathrm{m} / \mathrm{z} 295$ which is the base peak and M+2 peaks at 297.

Condensation of hydrazide with various substituted aromatic carboxylic acid followed by cyclization in presence of phosphorous oxychloride under reflux gave the respective 1,3,4-oxadiazole derivatives (4a-r) (Table-1). The evidence for the formation of 1,3,4-oxadiazole derivatives were obtained by recording the proton NMR and from FAB mass spectra.

The ${ }^{1} \mathrm{H}$ NMR spectrum of $\mathbf{4}$, the methyl protons came into resonance as a singlet at $\delta 2.44$ integrating for three protons while the singlet due to $-\mathrm{O}-\mathrm{CH}_{2}$ - came into resonance at $\delta 5.43$ accounting for 2 protons. The signal for naphthalene ring proton appeared as follows $\mathrm{C}_{4}-\mathrm{H}$ appeared at $\delta 7.14$ as doublet $\mathrm{J}=7.5 \mathrm{~Hz}, \mathrm{C}_{1}-\mathrm{H}$ at 7.32 singlet, $\mathrm{C}_{8}-\mathrm{H}$ proton at $\delta 7.53$ $\mathrm{J}=8.7 \mathrm{~Hz}, \mathrm{C}_{3}-\mathrm{H}$ at $\delta 7.64 \mathrm{~J}=8.7 \mathrm{~Hz}, \mathrm{C}_{7}-\mathrm{H}$ at $\delta 7.70 \mathrm{~J}=8.9 \mathrm{~Hz}$ as doublets respectively and $\mathrm{C}_{5}-\mathrm{H}$ as singlet at $\delta 7.94$. The phenyl protons resonated at $\delta 7.41-7.37$ as a multiplet accounting 2 protons. The $\mathrm{C}_{4}-\mathrm{H}$ phenyl ring proton resonated at $\delta 7.86 \mathrm{~J}=6.8 \mathrm{~Hz}$ as a doublet and at $\delta 7.90$ as a singlet is due to $\mathrm{C}_{2}-\mathrm{H}$ proton. The $\mathrm{FAB}$ mass of this compound showed the molecular ion peak at $\mathrm{m} / \mathrm{z}=395$, which is also the base peak there by indicating the stability of oxadiazole derivative. The other peak was observed at $397(\mathrm{M}+2)$ indicating the presence of bromine.

Compound 5-\{[(6-bromo-2-naphthyl)oxy]methyl\}-1,3,4-oxadiazole-2(3H)-thione (5) was synthesized by the ring closure reaction of hydrazide (3) with carbon disulfide in presence of a base. A series of Mannich bases (6a-j) were then synthesized by the reaction of (5) with suitable primary / secondary amines and formaldehyde in methanol. The compound (5) was also treated with alkyl halide in alkaline media to get 2-\{[(6-bromo-2-naphthyl)oxy]methyl\}-5-(alkylthio)-1,3,4-oxadiazole (7a-b) and with substituted benzylchlorides in presence of potassium carbonate to get (7c-g). The structure of newly synthesized compounds were established on the basis of elemental analysis and spectral (IR, ${ }^{1} \mathrm{H}$ NMR, and FAB mass) data.

5-\{[(6-Bromo-2-naphthyl)oxy]methyl\}-1,3,4-oxadiazole-2(3H)-thione (5) was obtained by reacting hydrazide (3) with carbon disulfide in presence of $\mathrm{KOH}$ in methanolic medium under reflux condition followed by acidification with $\mathrm{HCl}$. The evidence for the proposed structure was obtained by recording the IR, proton NMR and FAB mass.

The IR spectra showed a band in the region of $3400 \mathrm{~cm}^{-1}$ characteristic of NH group and at $2950 \mathrm{~cm}^{-1}$ of C-H stretching. The $\mathrm{C}=\mathrm{S}$ stretching was observed at $1255 \mathrm{~cm}^{-1}$ and $\mathrm{C}=\mathrm{N}$ at $1635 \mathrm{~cm}^{-1}$. In the ${ }^{1} \mathrm{H} \mathrm{NMR}$ spectra the $\mathrm{O}-\mathrm{CH}_{2}$ - proton came into resonance at $\delta 5.37$ as singlet. The $\mathrm{C}_{4}-\mathrm{H}$ proton of naphthalene ring resonated as doublet at $\delta 7.29$ with $\mathrm{J}=7.1 \mathrm{~Hz}$, $\mathrm{C}_{8}-\mathrm{H}$ at $\delta 7.42$ as doublet with $\mathrm{J}=7.1 \mathrm{~Hz}, \mathrm{C}_{1}-\mathrm{H}$ as singlet at $\delta 7.52, \mathrm{C}_{3}-\mathrm{H}$ resonated as doublet at $\delta 7.78 \mathrm{with} \mathrm{J}=8.85 \mathrm{~Hz}$, $\mathrm{C}_{7}-\mathrm{H}$ at $\delta 7.87$ as doublet with $\mathrm{J}=8.82 \mathrm{~Hz}$. The $\mathrm{C}_{5}-\mathrm{H}$ proton appeared as singlet at $\delta 8.15$ and a broad singlet at $\delta 15.1$ for SH proton. The mass spectrum of this compound showed molecular ion peak at m/z: 337 and $\mathrm{M}+2$ at 339 .

Further the Mannich reaction of oxadiazole (5) with primary/secondary amine and formaldehyde in methanol medium gave corresponding N-Mannich base (6a-j). The Mannich bases were confirmed by analytical and spectral studies.

The ${ }^{1} \mathrm{H}$ NMR spectrum of Mannich base $\mathbf{6 a}\left(\mathrm{CDCl}_{3}\right)$, the morpholine ring protons appeared as triplet at $\delta 2.77$ and 3.67 accounting for 4 protons each. The singlet due to $\mathrm{O}_{-} \mathrm{CH}_{2}$ - appeared at 4.99 integrating for two protons, while the $-\mathrm{N}^{-} \mathrm{CH}_{2}$ - protons resonated as singlet at $\delta 5.15$. The $\mathrm{C}_{7}-\mathrm{H}$ naphthalene ring proton resonated as doublet at $\delta 7.7$ with $\mathrm{J}=9.0 \mathrm{~Hz}$ and $\mathrm{C}_{5}-\mathrm{H}$ proton resonated at $\delta 7.95$ as singlet. The compound $\mathbf{6 a}$ is also confirmed with single crystal X-ray study, which confirms the formation of N-Mannich (Jasinski, Yathirajan et al., 2009)

Condensation of 5-\{[(6-bromo-2-naphthyl)oxy]methyl $\}$-1,3,4-oxadiazole-2(3H)-thione (5) with alkyl halide in ethanol by sonicating in presence of $10 \% \mathrm{NaOH}$ gave $\mathbf{7 a - b}$. The ${ }^{1} \mathrm{H}$ NMR spectrum of S-methylated oxadiazole $7 \mathbf{a}$ showed a singlet at $\delta 2.73$ accounting for three protons for $\mathrm{SCH}_{3}$ and the $\mathrm{O}_{-} \mathrm{CH}_{2}$ - protons resonated at $\delta 5.33$ as singlet. The proton $\mathrm{C}_{4}-\mathrm{H}$ and $\mathrm{C}_{1}-\mathrm{H}$ proton appeared as multiplet at $\delta 7.20-7.24, \mathrm{C}_{8}-\mathrm{H}$ at $\delta 7.52$ as doublet with $\mathrm{J}=8.7 \mathrm{~Hz}, \mathrm{C}_{3}-\mathrm{H}$ at $\delta$ 7.62 as doublet with $\mathrm{J}=8.7 \mathrm{~Hz}, \mathrm{C}_{7}-\mathrm{H}$ at $\delta 7.67$ as doublet $\mathrm{J}=8.9 \mathrm{~Hz}$ and $\mathrm{C}_{5}-\mathrm{H}$ at $\delta 7.93$ as singlet. The $\mathrm{FAB}$ mass of this compound showed the molecular ion peak at $\mathrm{m} / \mathrm{z}=351$. 
Further reacting (5) with substituted benzyl chloride in presence of potassium carbonate yielded 7c-g. In a typical example, the ${ }^{1} \mathrm{H}$ NMR spectrum of 7f, the methyl protons appeared as singlet at $\delta 2.31$ integrating for three protons. The signal due to $\mathrm{S}-\mathrm{CH}_{2}$ protons appeared at $\delta 4.43$ integrating for two protons while the $\mathrm{O}-\mathrm{CH}_{2}$ protons resonated as singlet at $\delta 5.30$ integrating for two protons. The phenyl ring protons resonated as multiplates at $\delta$ 7.15-7.25. The $\mathrm{C}_{3}-\mathrm{H}, \mathrm{C}_{8}-\mathrm{H}$, $\mathrm{C}_{7}-\mathrm{H}$ protons of naphthalene ring resonated as doublets at $\delta 7.51,7.60$ and 7.65 respectively with $\mathrm{J}=9 \mathrm{~Hz}$. The $\mathrm{C}_{5}-\mathrm{H}$ proton resonated at $\delta 7.91$ as singlet. Further the mass spectrum of this compound showed molecular ion peak at $\mathrm{m} / \mathrm{z}$ 441.in agreement with the molecular formula $\mathrm{C}_{21} \mathrm{H}_{17} \mathrm{BrN}_{2} \mathrm{O}_{2} \mathrm{~S}$. The peak at $\mathrm{m} / \mathrm{z} 443(\mathrm{M}+2)$ is due to presence of bromine atom in the molecule.

All the newly synthesized compounds were screened for their antimicrobial activity. The investigation of antibacterial and antifungal screening data revealed that all the tested compounds (4a-r) and (6a-j) showed moderate to good inhibition at $\mu \mathrm{g} \mathrm{ml}^{-1}$ in DMSO. The compounds $\mathbf{4 p}$ and $\mathbf{4 r}$ showed comparatively good activity against all the bacterial strains. The good activity is attributed to the presence of pharmacologically active group attached to phenyl group at position $\mathbf{5}$ of the oxadiazole ring. Introduction of aryl moiety carrying chloro and dichloro group enhanced activity compared to the standard against T. mentagrophytes, A. flavus and A. fumigatus. The presence of N-Mannich base has shown good antibacterial and antifungal activity. Among the tested compounds, Mannich bases $\mathbf{6 a}, \mathbf{6 b}, \mathbf{6 f}, \mathbf{6 g}$, and $\mathbf{6 h}$ have shown remarkable activity against all tested microorganisms. This may be attributed to the presence of pharmacologically active morpholine, 4-methylpiperazine, 2-fluorophenyl, 4-chlorophenyl and 2-methylphenyl groups associated with oxadiazole ring, while S-methylation caused decrease in activity against most of the strains. The compounds $7 \mathbf{c}-\mathbf{g}$ were inactive compared to that of standard against all the bacterial and fungal strains. Results of antibacterial and antifungal screening are discussed in Table-4 and Table-5 respectively.

\section{Conclusion}

The research study reports the successful synthesis and antimicrobial activity of new 1,3,4 oxadiazole derivatives bearing 6-bromonaphthalene moiety. The antimicrobial activity study revealed that all the compounds tested showed moderate to good antibacterial and antifungal activities against tested pathogenic strains. Compounds with phenyl ring substituted with chloro and dichloro showed good activity. Mannich base with morpholine, 4-methylpiperazine, phenyl ring substituted with 2-fluro, 4-chloro and 2-methyl group showed good activity where as (aryl)thio groups were inactive.

\section{Acknowledgement}

One of the authors (ANM) is thankful to University of Mysore for research facilities and SeQuent Scientific Ltd for permitting to undertake research. The authors are also thankful to the Head, SAIF, CDRI, Lucknow for the spectral data.

\section{References}

Aboraia, A.S., Abdel-Rahman, H.M., Mahfouz, N.M., EL-Gendy, M.A. (2006). Novel 5-(2-hydroxyphenyl)-3-substituted-2,3-dihydro-1,3,4-oxadiazole-2-thione derivatives: Promising anticancer agents. Bioorganic and Medicinal Chemistry, 14: 1236-1246.

Adelstein, G. W., Yen, C. H., Dajani, E. Z., Bianchi, R. G. (1976). 3,3-Diphenyl-3-(2-alkyl-1,3,4-oxadiazol-5-yl)propylcycloalkylamines, a novel series of antidiarrheal agents. Journal of Medicinal Chemistry, 19: 1221-1225.

Ali, M.A., Shaharyar, M. (2007). Oxadiazole mannich bases: Synthesis and antimycobacterial activity. Bioorganic and Medicinal Chemistry, 17: 3314-3316.

Amir, M., Shahani, S. (1998). Synthesis and anti-inflammatory of naphthylmethyl oxadiazoles, thiadiazoles and triazoles. Indian journal of Heterocyclic Chemistry, 8:107-110.

Arthington-Skaggs, B. A., Motley, M., Warnock, D.W., Morrison, C.J. (2000). Comparitive Evaluation of PASCO and National Committee for Clinical laboratory standards M27-A broath micro dilution methods for antifungal drug susceptibility testing of yeasts. Journal of Clinical Microbiology, 38: 2254-2260.

Barry, A. L. (1991). Procedure for testing antimicrobial agents in agar media, in:VL Corian (Ed), Antibiotics in Laboratory Medicine, Williams and Wilkins, Baltimore, MD, 1-16.

Boschelli, D. H., Connor, D. T., Bornemeier, D. A., Dyer, R. D., Kennedy, J. A., Kuipers, P. J., Okonkwo, G. C., Svhrier, D. J., Wright, C.D. (1993). 1,3,4-Oxadiazole, 1,3,4-thiadiazole, and 1,2,4-triazole analogs of the fenamates: in vitro inhibition of cyclooxygenase and 5-lipoxygenase activities. Journal of Medicinal Chemistry, 36:1802-1810.

Chen, H., Li, Z., Han, Y. (2000). Synthesis and Fungicidal Activity against Rhizoctonia solani of 2-Alkyl (Alkylthio)-5-pyrazolyl-1,3,4-oxadiazoles (Thiadiazoles). Journal of Agricultural and Food Chemistry, 48: 5312-5315. 
Dimmock, J. R., Kumar, P. (1997). Anticancer and Cytotoxic Properties of Mannich Bases. Current Medicinal Chemistry, 4: 1-22.

Fenlon, C. H., Cynamon, M. H. (1986). Comparative in vitro activities of ciprofloxacin and other 4-quinolones against Mycobacterium tuberculosis and Mycobacterium intracellulare. Antimicrobial Agents and Chemotherapy, 29: $386-388$.

Hashem, A. I., Youssef, A. S. A., Kandeel, K. A., Abou-Elmagd, W. S. I. (2007). Conversion of some 2(3H)-furanones bearing a pyrazolyl group into other heterocyclic systems with a study of their antiviral activity. European Journal of Medicinal Chemistry, 42: 934-939.

Hokfelt, B., Jonsson, A. (1962). Hypoglycemic Activity in Relation to Chemical Structure of Potential Oral Antidiabetic Substances. III. 2-Benzenesulfonamido-5-alkyl-1,3,4-thiadiazoles and -oxadiazoles. Journal of Medicinal Chemistry, 5: 247-257.

James, D., Lowry, M., Jaqua, M. J., Selepak, S. T. (1970). Detailed Methodology and Implementation of a Semi automated Serial Dilution Micro technique for Antimicrobial Susceptibility Testing. Applied Microbiology, 20 : 46-53.

Jasinski, J. P., Butcher, R. J., Mayekar, A. N., Yathirajan, H. S., Narayana, B., Sarojini, B. K. (2009). Synthesis and Crystal Structure of 5-[2-(6-bromonaphthalenyloxymethyl)]3-(4-morpholinomethyl)-1,3,4-oxadiazole-2(3H)-thione. Journal of Chemical Crystallography, 39: 761-765.

Kishore, V., Kumar, S., Parmar, S. S., Stenberg, V. I. (1975). Anti-inflammatory and Antiproteolytic properties of naphthylthiosemicarbazides and cyclized oxadiazoles. Research Communications in Chemical Pathology and Pharmacology,11: 581-594.

Ke, S., Li, Z., Qian, X. (2008). 1,3,4-Oxadiazole-3(2H)-carboxamide derivatives as potential novel class of monoamine oxidase (MAO) inhibitors: Synthesis, evaluation, and role of urea moiety Bioorganic and Medicinal Chemistry,16(16): $7565-7572$.

Ke, S., Liu, F., Wang, N., Yang, Q., Qian, X. (2009). 1,3,4-Oxadiazoline derivatives as novel potential inhibitors targeting chitin biosynthesis: Design, synthesis and biological evaluation Bioorganic and Medicinal Chemistry Letters, 19 (2): 332-335.

Kotecka, B.M., Barlin, G.B., Edstein M.D., Rieckmann, K.H. (1997). New Quinoline Di-Mannich Base Compounds with Greater Antimalarial Activity than Chloroquine, Amodiaquine or Pyronaridine. Antimicrobial Agents and Chemotherapy, 41(6): 1369-1374.

Macaev, F., Rusu, G., Pogrebnoi, S., Gudima, A., Stingaci, E., Vlad, L., Shvets, N., Kandemirli, F., Dimoglo, A., Reynolds, R. (2005). Synthesis of novel 5-aryl-2-thio-1,3,4-oxadiazoles and the study of their structure-anti-mycobacterial activities. Bioorganic and Medicinal Chemistry, 13: 4842-4850.

Narayana, B., Vijaya Raj, K. K., Ashalatha, B. V., Suchetha Kumari, N. (2005). Synthesis of Some New 2-(6-Methoxy-2-Naphthyl)-5-Aryl-1,3,4-Oxadiazoles as Possible Non-steroidal Anti-inflammatory and Analgesic Agents, Archive der Pharmazie - Chemistry in Life Sciences, 338: 373-377.

Rajak, H., Kharya, M.D., Mishra, P. (2007). Synthesis of some novel oxadiazole and oxadiazoline analogue for their anti-inflammatory activity. Journal of Pharmaceutical Society of Japan (Yakugaku Zasshi), 127(10): 1757-1764.

Sarojini, B.K., Narayana, B., Mayekar, A. N., Yathirajan, H. S., Bolte, M. (2007). Ethyl (6-bromo-2-naphthyloxy)acetate, Acta Crystallogrphica, E63: o4447.

Sridhar, S. K., Pandeya, S. N., Stables, J. P., Ramesh, A. (2002). Anticonvulsant activity of hydrazones, Schiff and Mannich bases of isatin derivatives. European Journal of Pharmaceutical Sciences, 16:129-132.

Sriram. D., Banerjee D., Yogeeshwari. P. (2009). Efavirenz Mannich bases: Synthesis, anti-HIV and antitubercular activities. Journal of Enzyme Inhibition and Medicinal Chemistry, 24: 1-5

Verma, R. S.,Khan, Z. K., Singh, A. P. (1998). (Ed), Antifungal Agents: Past, Present and Future Prospects, National Academy of Chemistry and Biology, Lucknow, India, 55-128.

Yale, H. L., Losee, K. (1966). 2-Amino-5-Substituted 1,3,4-Oxadiazoles and 5-Imino-2-Substituted 1,3,4-Oxadiazolines. A Group of Novel Muscle Relaxants. Journal of Medicinal Chemistry, 9: 478-483.

Zarghi, A., Hajimahdi, Z., Mohebbi, S., Rashidi, H., Mozaffari, S., Sarraf, S., Faizi, M., Tabatabaee, S. A. (2008). Design and Synthesis of New 2-Substituted-5-[2-(2-halobenzyloxy)phenyl]-1,3,4-oxadiazoles as Anticonvulsant Agents. Chemical and Pharmaceutical Bulletin, 56(4): 509-512. 
<smiles>CCOC(=O)COc1ccc2cc(Br)ccc2c1</smiles>

(1)
(2)

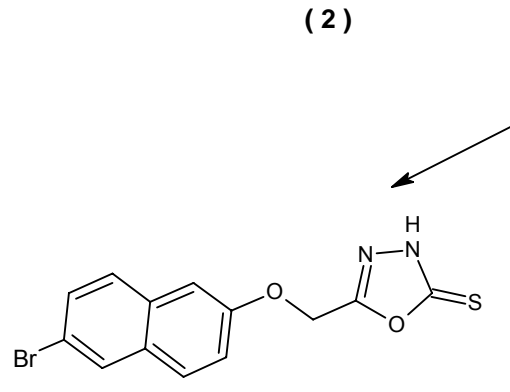

( 5 )

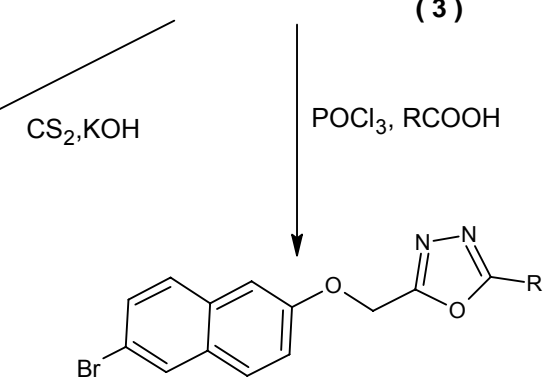

(4)

Scheme 1. Synthesis of 2-\{[(6-bromo-2-naphthyl)oxy]methyl $\}$-5-aryl-1,3,4-oxadiazole

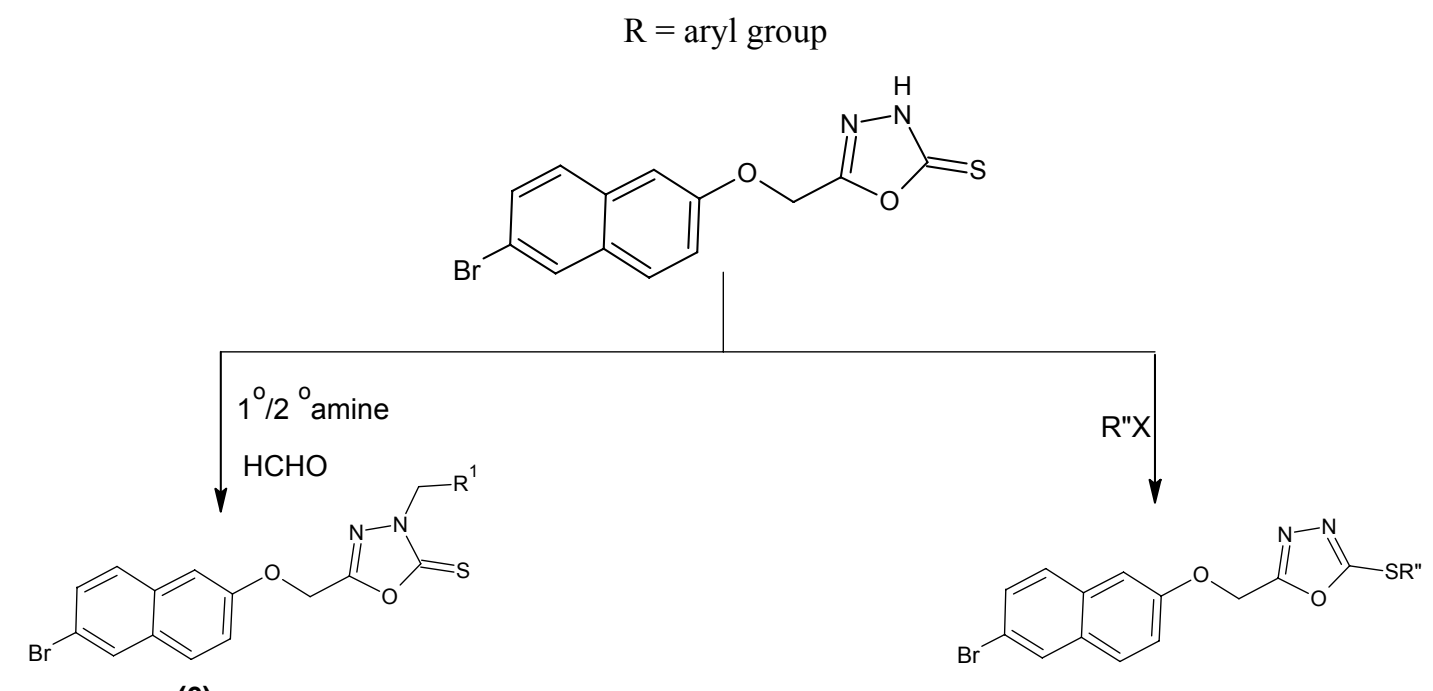

(6)

(7)

Scheme 2. Synthesis of Mannich bases and 2-\{[(6-bromo-2-naphthyl)oxy]methyl $\}-5-[($ alkyl/aryl)thio]-1,3,4-oxadiazole $\mathrm{R}^{1}=$ primary/secondary amine

$\mathrm{R}^{11}=\mathrm{CH}_{3}, \mathrm{C}_{2} \mathrm{H}_{5}$, substituted benzyl 
Table 1. Characterization data of compounds (4a-r)

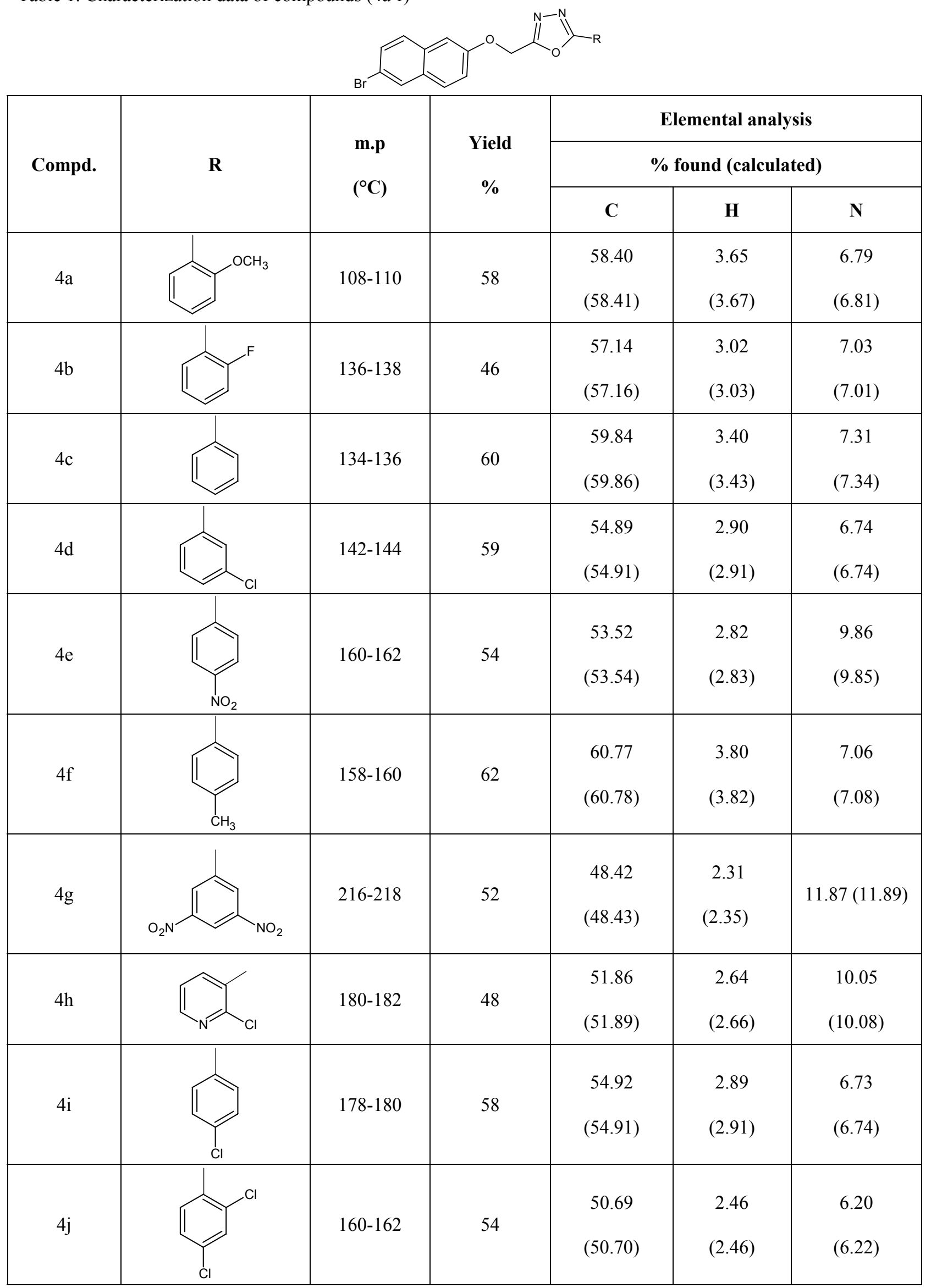




\begin{tabular}{|c|c|c|c|c|c|c|}
\hline $4 k$ & & 106-108 & 50 & $\begin{array}{c}57.56 \\
(57.59)\end{array}$ & $\begin{array}{c}3.54 \\
(3.56)\end{array}$ & $\begin{array}{c}10.61 \\
(10.60)\end{array}$ \\
\hline 41 & & $110-112$ & 63 & $\begin{array}{c}57.71 \\
(57.73)\end{array}$ & $\begin{array}{c}3.04 \\
(3.06)\end{array}$ & $\begin{array}{c}7.06 \\
(7.08)\end{array}$ \\
\hline $4 m$ & & $180-182$ & 56 & $\begin{array}{c}50.71 \\
(50.70)\end{array}$ & $\begin{array}{c}2.44 \\
(2.46)\end{array}$ & $\begin{array}{c}6.20 \\
(6.22)\end{array}$ \\
\hline $4 n$ & & $164-166$ & 52 & $\begin{array}{c}56.53 \\
(56.56)\end{array}$ & $\begin{array}{c}3.14 \\
(3.16)\end{array}$ & $\begin{array}{r}10.97 \\
(10.99)\end{array}$ \\
\hline 40 & & $150-152$ & 60 & $\begin{array}{c}63.15 \\
(63.17)\end{array}$ & $\begin{array}{r}4.01 \\
(4.02)\end{array}$ & $\begin{array}{c}5.87 \\
(5.89)\end{array}$ \\
\hline $4 p$ & & $150-152$ & 54 & $\begin{array}{r}54.90 \\
(54.91)\end{array}$ & $\begin{array}{c}2.92 \\
(2.91)\end{array}$ & $\begin{array}{c}6.72 \\
(6.74)\end{array}$ \\
\hline $4 q$ & & $146-148$ & 56 & $\begin{array}{c}50.69 \\
(50.70)\end{array}$ & $\begin{array}{c}2.45 \\
(2.46)\end{array}$ & $\begin{array}{c}6.20 \\
(6.22)\end{array}$ \\
\hline $4 r$ & & $134-136$ & 50 & $\begin{array}{c}50.71 \\
(50.70)\end{array}$ & $\begin{array}{c}2.46 \\
(2.46)\end{array}$ & $\begin{array}{c}6.21 \\
(6.22)\end{array}$ \\
\hline
\end{tabular}


Table 2. Characterization data of compounds $6 a-j$

\begin{tabular}{|c|c|c|c|c|c|c|}
\hline \multirow{3}{*}{ Compd. } & \multirow{3}{*}{$\mathbf{R}^{1}$} & \multirow{3}{*}{$\begin{array}{l}\text { m.p } \\
\left({ }^{\circ} \mathrm{C}\right)\end{array}$} & \multirow{3}{*}{$\begin{array}{c}\text { Yield } \\
\%\end{array}$} & \multirow{2}{*}{\multicolumn{3}{|c|}{$\begin{array}{c}\text { Elemental analysis } \\
\text { \% found (calculated) }\end{array}$}} \\
\hline & & & & & & \\
\hline & & & & C & $\mathbf{H}$ & $\mathbf{N}$ \\
\hline $6 a$ & & $110-112$ & 73 & $\begin{array}{c}49.50 \\
(49.55)\end{array}$ & $\begin{array}{c}4.13 \\
(4.16)\end{array}$ & $\begin{array}{c}9.59 \\
(9.63)\end{array}$ \\
\hline $6 b$ & & $182-184$ & 56 & $\begin{array}{c}50.70 \\
(50.78)\end{array}$ & $\begin{array}{r}4.69 \\
(4.71)\end{array}$ & $\begin{array}{r}12.41 \\
(12.46)\end{array}$ \\
\hline $6 c$ & & $110-112$ & 42 & $\begin{array}{c}52.51 \\
(52.54)\end{array}$ & $\begin{array}{c}4.61 \\
(4.64)\end{array}$ & $\begin{array}{c}9.63 \\
(9.67)\end{array}$ \\
\hline $6 \mathrm{~d}$ & & $150-152$ & 50 & $\begin{array}{c}51.16 \\
(51.19)\end{array}$ & $\begin{array}{r}4.72 \\
(4.77)\end{array}$ & $\begin{array}{c}9.90 \\
(9.94)\end{array}$ \\
\hline $6 e$ & 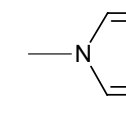 & $162-164$ & 51 & $\begin{array}{c}48.61 \\
(48.93)\end{array}$ & $\begin{array}{c}3.11 \\
(3.14)\end{array}$ & $\begin{array}{r}13.39 \\
(13.42)\end{array}$ \\
\hline $6 f$ & & $152-154$ & 58 & $\begin{array}{c}52.16 \\
(52.18)\end{array}$ & $\begin{array}{c}3.29 \\
(3.28)\end{array}$ & $\begin{array}{c}9.09 \\
(9.12)\end{array}$ \\
\hline $6 \mathrm{~g}$ & & $146-148$ & 48 & $\begin{array}{c}50.36 \\
(50.38)\end{array}$ & $\begin{array}{c}3.17 \\
(3.17)\end{array}$ & $\begin{array}{r}8.80 \\
(8.81)\end{array}$ \\
\hline $6 \mathrm{~h}$ & & $162-164$ & 50 & $\begin{array}{c}55.23 \\
(55.27)\end{array}$ & $\begin{array}{c}3.91 \\
(3.97)\end{array}$ & $\begin{array}{r}9.18 \\
(9.20)\end{array}$ \\
\hline $6 \mathrm{i}$ & & $180-182$ & 58 & $\begin{array}{c}46.04 \\
(46.08)\end{array}$ & $\begin{array}{c}2.88 \\
(2.90)\end{array}$ & $\begin{array}{c}8.01 \\
(8.06)\end{array}$ \\
\hline $6 j$ & $-\mathrm{NH}$ & $152-154$ & 51 & $\begin{array}{c}53.31 \\
(53.39)\end{array}$ & $\begin{array}{c}3.80 \\
(3.84)\end{array}$ & $\begin{array}{c}8.81 \\
(8.89)\end{array}$ \\
\hline
\end{tabular}


Table 3. Characterization data of compounds 7a-g<smiles>[R7]Sc1nnc(COc2ccc3cc(Br)ccc3c2)o1</smiles>

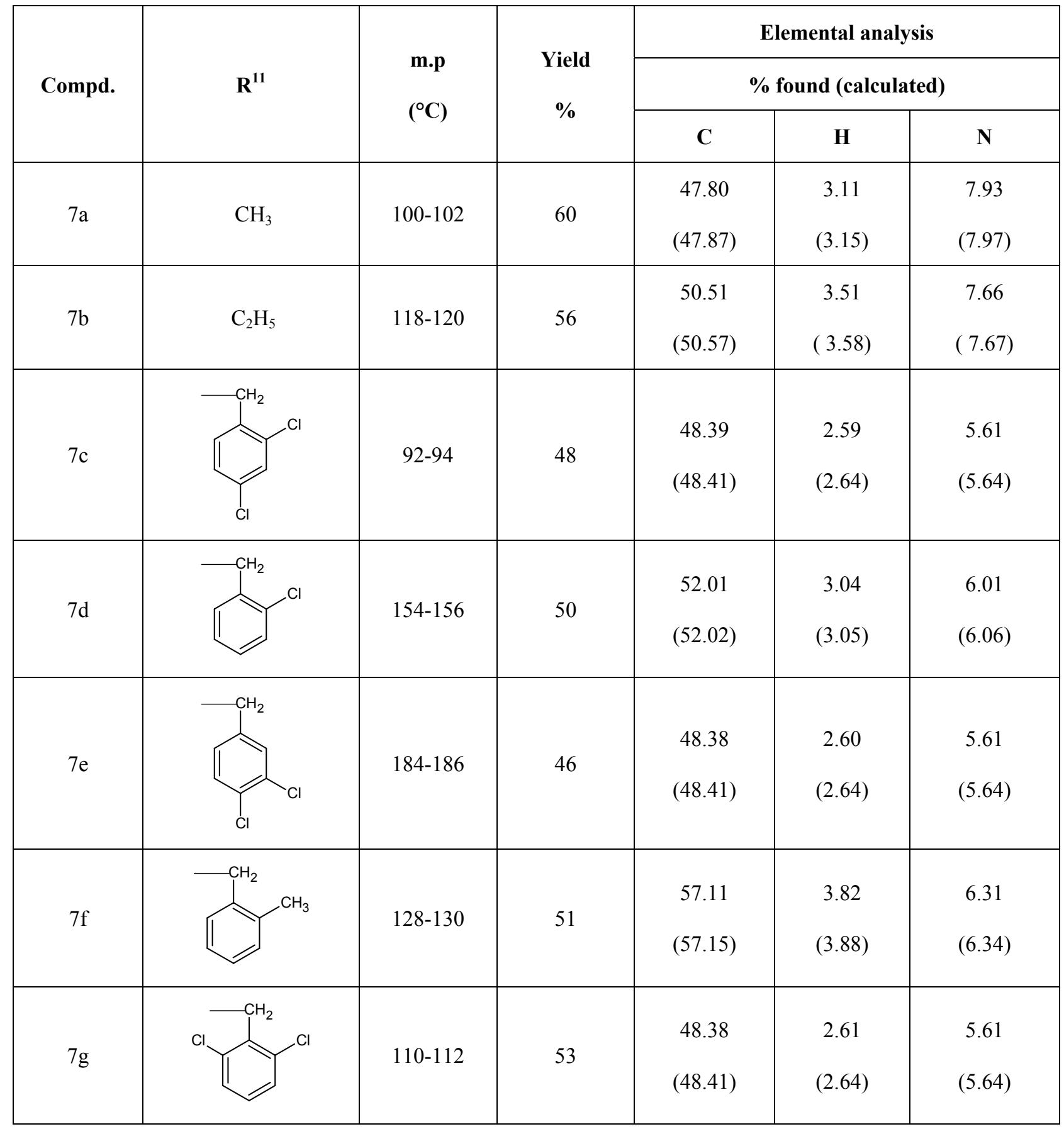


Table 4. Antibacterial activity of the compounds. MIC in $\mu \mathrm{gml}^{-1}$ (Zone of inhibition in $\mathrm{mm}$ )

\begin{tabular}{|c|c|c|c|c|}
\hline Compounds & E.Coli & Staphylococcus & Klebsiella & Pseudomonas \\
\hline $4 a$ & - & - & - & - \\
\hline $4 b$ & $12.5(11)$ & - & - & - \\
\hline $4 \mathrm{c}$ & - & - & - & - \\
\hline $4 d$ & $12.5(15)$ & $6.25(16)$ & - & $6.25(16)$ \\
\hline $4 \mathrm{e}$ & $12.5(10)$ & $6.25(12)$ & - & $6.25(12)$ \\
\hline $4 \mathrm{f}$ & $12.5(11)$ & - & - & $12.5(15)$ \\
\hline $4 \mathrm{~g}$ & $12.5(10)$ & & - & - \\
\hline $4 \mathrm{~h}$ & $12.5(10)$ & - & $6.25(12)$ & - \\
\hline $4 \mathrm{i}$ & $12.5(10)$ & - & - & - \\
\hline $4 j$ & $12.5(11)$ & $6.25(12)$ & - & - \\
\hline $4 \mathrm{k}$ & $12.5(10)$ & - & - & - \\
\hline 41 & - & - & - & - \\
\hline $4 \mathrm{~m}$ & $12.5(10)$ & & - & - \\
\hline $4 n$ & - & - & - & - \\
\hline 40 & $12.5(10)$ & & - & - \\
\hline $4 p$ & $6.25(20)$ & $6.25(15)$ & $6.25(18)$ & $6.25(12)$ \\
\hline $4 q$ & - & - & - & - \\
\hline $4 r$ & $6.25(18)$ & $6.25(18)$ & $6.25(15)$ & $6.25(18)$ \\
\hline $6 a$ & $6.25(18)$ & $6.25(20)$ & $6.25(18)$ & $6.25(18)$ \\
\hline $6 \mathrm{~b}$ & $12.5(15)$ & $12.5(15)$ & $12.5(18)$ & $12.5(15)$ \\
\hline $6 c$ & $12.5(10)$ & - & - & - \\
\hline $6 \mathrm{~d}$ & $12.5(14)$ & - & $6.25(12)$ & - \\
\hline $6 \mathrm{e}$ & $12.5(15)$ & - & $6.25(11)$ & - \\
\hline $6 f$ & $6.25(18)$ & $6.25(16)$ & $6.25(18)$ & $6.25(15)$ \\
\hline $6 \mathrm{~g}$ & $12.5(15)$ & $12.5(15)$ & $12.5(12)$ & $12.5(12)$ \\
\hline $6 \mathrm{~h}$ & $12.5(18)$ & $12.5(15)$ & $12.5(15)$ & $12.5(15)$ \\
\hline $6 \mathrm{i}$ & $12.5(11)$ & $12.5(14)$ & $12.5(12)$ & $12.5(12)$ \\
\hline $6 \mathrm{j}$ & $12.5(10)$ & $6.25(8)$ & - & - \\
\hline $7 \mathrm{a}$ & $12.5(8)$ & $12.5(10)$ & $12.5(10)$ & $12.5(10)$ \\
\hline $7 \mathrm{~b}$ & $12.5(10)$ & $12.5(10)$ & $12.5(12)$ & $12.5(12)$ \\
\hline $7 \mathrm{c}$ & - & - & - & - \\
\hline $7 \mathrm{~d}$ & - & - & - & - \\
\hline $7 \mathrm{e}$ & - & - & - & - \\
\hline $7 f$ & - & - & - & - \\
\hline $7 \mathrm{~g}$ & - & - & - & - \\
\hline Ciprofloxacin & $6.25(30)$ & $6.25(30)$ & $6.25(27)$ & $6.25(28)$ \\
\hline
\end{tabular}

Note: The MIC values were evaluated at concentration range, 1.5-12.5 $\mu \mathrm{g} / \mathrm{ml}$. The figures in the table show the MIC values in $\mu \mathrm{g} / \mathrm{ml}$ and the corresponding zone of inhibition in $\mathrm{mm}$. 
Table 5. Antifungal activity of the compounds. MIC in $\mu \mathrm{gml}^{-1}$ (Zone of inhibition in $\mathrm{mm}$ )

\begin{tabular}{|c|c|c|c|c|}
\hline Compounds & Penicillium & Trichophton & AS Flavus & $A S$ fumigatus \\
\hline $4 a$ & - & - & - & - \\
\hline $4 b$ & - & $12.5(11)$ & - & - \\
\hline $4 c$ & - & - & - & - \\
\hline $4 d$ & $6.25(12)$ & $12.5(15)$ & $6.25(15)$ & - \\
\hline $4 \mathrm{e}$ & $6.25(16)$ & $12.5(15)$ & $6.25(16)$ & - \\
\hline $4 \mathrm{f}$ & - & $12.5(18)$ & $12.5(18)$ & - \\
\hline $4 g$ & - & $12.5(16)$ & $12.5(15)$ & - \\
\hline $4 \mathrm{~h}$ & - & $12.5(16)$ & - & $6.25(15)$ \\
\hline $4 \mathrm{i}$ & - & $12.5(18)$ & - & - \\
\hline $4 \mathrm{j}$ & $6.25(16)$ & $12.5(18)$ & - & - \\
\hline $4 \mathrm{k}$ & - & $12.5(18)$ & - & - \\
\hline 41 & - & - & - & - \\
\hline $4 \mathrm{~m}$ & - & $12.5(15)$ & - & - \\
\hline $4 n$ & - & - & - & - \\
\hline 40 & - & $12.5(15)$ & - & - \\
\hline $4 \mathrm{p}$ & $6.25(18)$ & $6.25(18)$ & $6.25(15)$ & $6.25(18)$ \\
\hline $4 q$ & - & - & - & - \\
\hline $4 \mathrm{r}$ & $6.25(18)$ & $6.25(15)$ & $6.25(18)$ & $6.25(15)$ \\
\hline $6 \mathrm{a}$ & $6.25(18)$ & $6.25(18)$ & $6.25(20)$ & $6.25(20)$ \\
\hline $6 \mathrm{~b}$ & $12.5(15)$ & $12.5(11)$ & $12.5(15)$ & $12.5(10)$ \\
\hline $6 c$ & $12.5(10)$ & - & - & - \\
\hline $6 \mathrm{~d}$ & $12.5(15)$ & - & $12.5(16)$ & \\
\hline $6 e$ & $12.5(12)$ & - & $12.5(16)$ & \\
\hline $6 f$ & $6.25(16)$ & $6.25(18)$ & $6.25(16)$ & $6.25(16)$ \\
\hline $6 \mathrm{~g}$ & $12.5(18)$ & $12.5(15)$ & $12.5(20)$ & $12.5(18)$ \\
\hline $6 \mathrm{~h}$ & $6.25(20)$ & $6.25(15)$ & $6.25(15)$ & $6.25(18)$ \\
\hline $6 \mathrm{i}$ & $6.25(16)$ & $12.5(18)$ & $12.5(11)$ & $12.5(15)$ \\
\hline $6 \mathrm{j}$ & $6.25(16)$ & $12.5(15)$ & $12.5(15)$ & $12.5(15)$ \\
\hline $7 \mathrm{a}$ & $12.5(10)$ & $12.5(9)$ & $12.5(10)$ & $12.5(10)$ \\
\hline $7 \mathrm{~b}$ & $12.5(10)$ & $12.5(10)$ & $12.5(10)$ & $12.5(10)$ \\
\hline $7 \mathrm{c}$ & - & - & - & - \\
\hline $7 \mathrm{~d}$ & - & - & - & - \\
\hline $7 \mathrm{e}$ & - & - & - & - \\
\hline $7 \mathrm{f}$ & - & - & - & - \\
\hline $7 \mathrm{~g}$ & - & - & - & - \\
\hline Cyclopiroxolamine & $6.25(27)$ & $3.12(30)$ & $3.125(28)$ & $6.25(28)$ \\
\hline
\end{tabular}

Note: The MIC values were evaluated at concentration range, 1.5-12.5 $\mu \mathrm{g} / \mathrm{ml}$. The figures in the table show the MIC values in $\mu \mathrm{g} / \mathrm{ml}$ and the corresponding zone of inhibition in $\mathrm{mm}$. 